\title{
Propagation of Natural Waves on Plates of a Variable Cross Section
}

\author{
Ismail Ibrahimovich Safarov, Zafar Ihterovich Boltaev \\ Bukhara Technological-Institute of Engineering, Bukhara, Republic of Uzbekistan \\ Email: safarov54@mail.ru
}

How to cite this paper: Safarov, I.I. and Boltaev, Z.I. (2018) Propagation of Natural Waves on Plates of a Variable Cross Section. Open Access Library Journal, 5: e4262. https://doi.org/10.4236/oalib.1104262

Received: December 15, 2017

Accepted: February 6, 2018

Published: February 9, 2018

Copyright (C) 2018 by authors and Open Access Library Inc.

This work is licensed under the Creative Commons Attribution International License (CC BY 4.0).

http://creativecommons.org/licenses/by/4.0/

\begin{abstract}
In this paper, a conjugate spectral problem and biorthogonality conditions for the problem of extended plates of variable thickness are constructed. A technique for solving problems and numerical results on the propagation of waves in infinite extended viscoelastic plates of variable thickness is described. The viscous properties of the material are taken into account using the Voltaire integral operator. The investigation is carried out within the framework of the spatial theory of viscoelasticity. The technique is based on the separation of spatial variables and the formulation of a boundary value problem for Eigen values which are solved by the Godunov orthogonal sweep method and the Muller method. Numerical values of the real and imaginary parts of the phase velocity are obtained depending on the wave numbers. In this case, the coincidence of numerical results with known data is obtained.
\end{abstract}

\section{Subject Areas}

Applied Physics, Continuum Mechanics

\section{Keywords}

Waveguide, Spectral Problem, Plane Wave Biorthogonality, Plastic, Dual Problem

\section{Introduction}

The study of the propagation of deformation waves in elastic and viscoelastic media is an important direction in modern wave dynamics. The main problem is the study of the dissipative (damping) properties of the system as a whole, as well as its stress-strain state. With the free propagation of waves, the dissipation reduces to the attenuation of free waves. The rate of damping quantitatively estimates the dissipative properties of the system: the greater the decay rate, the 
higher the dissipation [1] [2]. It is known [3] [4] that the normal waves in the deformed layer (Lamb waves) are not orthogonal in layer thickness, that is, the integral of the scalar product of displacement vectors of two different waves considered as a function of the coordinate perpendicular to the surfaces of the layer is not zero. They are also not orthogonal to the conjugate waves obtained from considering the adjoint problem. This circumstance introduces additional difficulties in solving practical problems [5] [6]. In this work, the difference from the known ones, the conjugate spectral problem, the biorthogonality conditions are constructed, an algorithm is developed, and numerical results are obtained for the problem of extended plates of variable thickness.

\section{The Mathematical Formulation of the Problem}

We consider the visco elastic waveguide as an infinite axial $x_{1}$ variable thickness (Figure 1). Basic relations of the classical theory of plates of variable thickness can be obtained on the basis of the principles of virtual displacements. The variation equation problem visco elasticity theory in three-dimensional statement has the form

$$
\iint_{V}\left(\sigma_{i j} \delta \varepsilon_{i j}+\rho u_{i} \delta u_{i}\right) \mathrm{d} x_{3} \mathrm{~d} x_{2} \mathrm{~d} x_{1}=0 \quad(i=1,2,3 ; j=1,2,3)
$$

where $\rho$-material density; $u_{i}$-displacement components; $\sigma_{i j}$ and $\varepsilon_{i j}$-components of the stress tensor and strain; $h$-plate thickness; $V$-the volume occupied by the body. In accordance with the hypotheses of Kirchhoff-Love

$$
\sigma_{12}=\sigma_{23}=\sigma_{33}=0, u_{i}=-x_{3} \frac{\partial w}{\partial x_{i}}, w\left(x_{3}, t\right)=w .
$$

Neglecting in (1) the members of which take into account the inertia of rotation normal to the middle plane, will have the following variation equation:

$$
\int_{S} \mathrm{~d} s \int_{-\frac{h}{2}}^{\frac{h}{2}}\left(\sigma_{11} \delta \varepsilon_{11}+2 \tau_{12} \delta \varepsilon_{12}+\tau_{22} \delta \varepsilon_{22}\right) \mathrm{d} x_{3}+\int_{S} \mathrm{~d} s \int_{-\frac{h}{2}}^{\frac{h}{2}} \rho \frac{\partial^{2} w}{\partial t^{2}} \delta w \mathrm{~d} x_{3}=0
$$

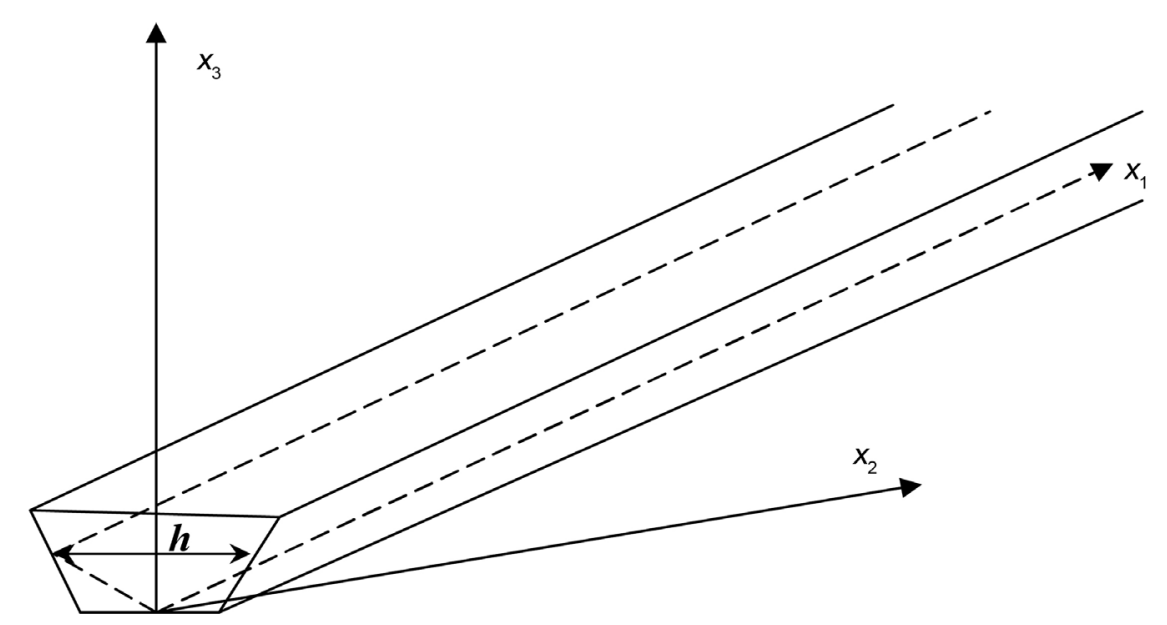

Figure 1. Design scheme: plates of variable thickness. 
Based on the geometric relationships and relations of the generalized Hooke's law, taking into account the kinematic hypotheses (2), the expressions for the components of the strain and stress tensor has the form [7]

$$
\begin{gathered}
\varepsilon_{i j}=\frac{1}{2}\left(\frac{\partial u_{i}}{\partial x_{j}}+\frac{\partial u_{j}}{\partial x_{i}}\right)-x_{3} \frac{\partial^{2} w}{\partial x_{i} \partial x_{j}}, \quad i, j=1,2 ; \\
\sigma_{11}=\frac{\tilde{E}}{1-v}\left(\varepsilon_{11}+v \varepsilon_{22}\right) ; \sigma_{22}=\frac{\tilde{E}}{1-v}\left(\varepsilon_{22}+v \varepsilon_{11}\right) ; \sigma_{12}=\frac{\tilde{E}}{1+v} \varepsilon_{12}, \\
\tilde{E}_{n} \varphi(t)=E_{0 n}\left[\varphi(t)-\int_{0}^{t} R_{E n}(t-\tau) \varphi(t) \mathrm{d} \tau\right],
\end{gathered}
$$

where $\varphi(t)$-arbitrary function of time; $v$-Poisson's ratio; $R_{E n}(t-\tau)$-the core of relaxation; $E_{01}$-instantaneous modulus of elasticity; we accept the integral terms in (5) small, then the function $\varphi(t)=\psi(t) \mathrm{e}^{-i \omega_{R} t}$, where $\psi(t)$-slowly varying function of time, $\omega_{R}$-real constant. Then, we replace of (6) approximate species [8]

$$
\bar{E}_{n} \varphi=E_{0 j}\left[1-\Gamma_{j}^{C}\left(\omega_{R}\right)-i \Gamma_{j}^{S}\left(\omega_{R}\right)\right] \varphi
$$

where $\Gamma_{n}^{C}\left(\omega_{R}\right)=\int_{0}^{\infty} R_{E n}(\tau) \cos \omega_{R} \tau \mathrm{d} \tau, \quad \Gamma_{n}^{S}\left(\omega_{R}\right)=\int_{0}^{\infty} R_{E n}(\tau) \sin \omega_{R} \tau \mathrm{d} \tau$, respectively, cosine and sine Fourier transforms relaxation kernel material. As an example, the visco elastic material take three parametric relaxation nucleus $R_{E n}(t)=A_{n} \mathrm{e}^{-\beta_{n} t} / t^{1-\alpha_{n}}$. Here $A_{n}, \alpha_{n}, \beta_{n}$-parameters relaxation nucleus. On the effect of the function $R_{E n}(t-\tau)$ superimposed usual requirements inerrability, continuity (except $t=\tau$ ), signs-certainty and monotony:

$$
R_{E n}>0, \frac{\mathrm{d} R_{E n}}{\mathrm{~d} t} \leq 0,0<\int_{0}^{\infty} R_{E n}(t) \mathrm{d} t<1 .
$$

Introducing the notation for points

$$
\begin{gathered}
M_{11}=\bar{D}\left(\frac{\partial^{2} w}{\partial x_{1}^{2}}+v \frac{\partial^{2} w}{\partial x_{2}^{2}}\right) ; M_{22}=\bar{D}\left(\frac{\partial^{2} w}{\partial x_{2}^{2}}+v \frac{\partial^{2} w}{\partial x_{1}^{2}}\right) ; \\
M_{12}=\bar{D}(1-v) \frac{\partial^{2} w}{\partial x_{1} \partial x_{2}}, \quad \bar{D}=\frac{\bar{E} h^{3}}{12\left(1-v^{2}\right)} .
\end{gathered}
$$

When $R_{E n}(t-\tau)=0$, then $D=\frac{E h^{3}}{12\left(1-v^{2}\right)}$. Here $E$ is the modulus of elasticity.

Integrating (3) in the strip thickness leads to the following form

$$
\int_{5}\left(M_{11} \frac{\partial^{2} \delta w}{\partial x_{1}^{2}}+2 M_{12} \frac{\partial^{2} \delta w}{\partial x_{1} \partial x_{2}}+M_{22} \frac{\partial^{2} \delta w}{\partial x_{2}^{2}}\right) \mathrm{d} s-\int_{s} \rho h \frac{\partial^{2} w}{\partial t^{2}} \delta w \mathrm{~d} s=0
$$

Integrating twice by parts and alignment to zero, the coefficients of variation $\delta w$ inside the body and on its boundary and we obtain the following differential equation 


$$
\frac{\partial^{2} M_{11}}{\partial x^{2}}+2 \frac{\partial^{2} M_{12}}{\partial x_{1} \partial x_{2}}+\frac{\partial^{2} M_{22}}{\partial x_{2}^{2}}+\rho h \ddot{w}=0,\left(\ddot{w}=\partial^{2} w / \partial t^{2}\right)
$$

with natural boundary conditions:

$$
\begin{aligned}
& \left\{\begin{array}{l}
\frac{\partial w}{\partial x_{2}}=0 \\
w=0 ; x_{2}=0 ; l_{2}
\end{array}\right. \\
& \left\{\begin{array}{l}
\frac{\partial w}{\partial x_{1}}=0 \\
w=0 ; x_{1}=0 ; l_{1}
\end{array}\right.
\end{aligned}
$$

The main alternative boundary conditions to them

$$
\begin{aligned}
& \left\{\begin{array}{l}
M_{22}=0 \\
\frac{\partial M_{22}}{\partial x_{2}}+2 \frac{\partial M_{12}}{\partial x_{1}}=0 ; x_{2}=0 ; l_{2}
\end{array}\right. \\
& \left\{\begin{array}{l}
M_{11}=0 \\
\frac{\partial M_{11}}{\partial x_{1}}+2 \frac{\partial M_{12}}{\partial x_{2}}=0 ; x_{1}=0 ; l_{1}
\end{array}\right.
\end{aligned}
$$

For, we construct a spectral problem by entering the following change of variables

$$
\begin{aligned}
& w=W ; \varphi=\frac{\partial W}{\partial x_{2}} ; \\
& M=\left(\frac{\partial^{2} W}{\partial x_{1}^{2}}+\frac{\partial^{2} W}{\partial x_{2}^{2}}\right) ; Q=\frac{\partial M_{22}}{\partial x_{2}}+\frac{\partial M_{12}}{\partial x_{1}} .
\end{aligned}
$$

Substituting (12) into (7) we obtain the differential equation of the system relatively sparse on the first derivatives $X_{2}$ :

$$
\begin{aligned}
& \frac{\partial Q}{\partial x_{2}}+\frac{\partial^{2} M}{\partial x_{1}^{2}}+\bar{D}^{\prime}(1-v) \frac{\partial^{2} \varphi}{\partial x_{1}^{2}}+\rho h \frac{\partial^{2} W}{\partial t^{2}}=0 ; \\
& \frac{\partial M}{\partial x_{2}}-Q-\bar{D}^{\prime \prime}(1-v) \frac{\partial^{2} W}{\partial x_{1}^{2}}=0 ; \\
& \bar{D} \frac{\partial \varphi}{\partial x_{2}}-M+\bar{D} \frac{\partial^{2} W}{\partial x_{1}^{2}}=0 ; \\
& \frac{\partial W}{\partial x_{2}}-\varphi=0 .
\end{aligned}
$$

And alternative boundary conditions $x_{2}=0 ; x_{2}=l_{2}$;

$$
\begin{gathered}
\varphi=0 \quad \text { or } \quad M-\bar{D}(1-v) \frac{\partial^{2} M}{\partial x_{1}^{2}}=0 ; \\
W=0 \text { or } Q+\bar{D}(1-v) \frac{\partial^{2} \varphi}{\partial x_{1}^{2}}=0 .
\end{gathered}
$$

and $x_{1}=0, x_{1}=l_{1}$, 


$$
\begin{aligned}
& \varphi=0 \quad \text { or } \quad M-\bar{D}(1-v) \frac{\partial^{2} M}{\partial x_{1}^{2}}=0 \\
& W=0 \text { or } Q+\bar{D}(1-v) \frac{\partial^{2} \varphi}{\partial x_{1}^{2}}=0
\end{aligned}
$$

Now consider the infinite along the axis $x_{1}$ band with an arbitrary thickness changes $h=h\left(x_{2}\right)$. We seek a solution of problem (13)-(15) in the for

$$
(Q, M, \varphi, W)^{\mathrm{T}}=(\bar{Q}, \bar{M}, \bar{\varphi}, \bar{W})^{\mathrm{T}} \mathrm{e}^{i\left(\alpha x_{1}-\omega t\right)}
$$

Describing the harmonic plane waves propagating along the axis $X_{1}$. Here $(\bar{Q}, \bar{M}, \bar{\varphi}, \bar{W})^{\mathrm{T}}$-complex amplitude-function; $k$-wave number; $C\left(C=C_{R}+i C_{i}\right)$ complex phase velocity; $\omega$-complex frequency.

To clarify their physical meaning, consider two cases:

1) $k=k_{R} ; C=C_{R}+i C_{i},\left(\omega_{R}=\omega_{I}+i \omega_{I}\right)$ then the solution of differential Equations (13) has the form of a sine wave at $X_{1}$, whose amplitude decays over time;

2) $k=k_{R}+i k_{I} ; C=C_{R}$, Then at each point $x_{1}$ fluctuations established, but $x_{1}$ attenuated.

In both cases, the imaginary part $k_{I}$ or $C_{I}$ characterized by the intensity of the dissipative processes. Substituting (16) in (17), we obtain a system of first order differential equations solved for the derivative

$$
\begin{aligned}
& \bar{Q}^{\prime}-\alpha^{2} \bar{M}-\alpha^{2} \bar{D}^{\prime}(1-v) \bar{\varphi}-\rho h \omega^{2} \bar{W}=0 ; \\
& \bar{M}^{\prime}-\bar{Q}+\alpha^{2} \bar{D}^{\prime}(1-v) \bar{W}=0 ; \\
& \bar{\varphi}^{\prime}-\frac{1}{\bar{D}} \bar{M}-\alpha^{2} \bar{W}=0 ; \\
& \bar{W}^{\prime}-\bar{\varphi}=0
\end{aligned}
$$

with boundary conditions at the ends of the band $x_{2}=0, l_{2}$, one of the four types

1) Swivel bearing: $\bar{W}=\bar{M}=0$

2) Sliding clamp: $\bar{Q}=\bar{\varphi}=0$

3) Anchorage: $\bar{W}=\bar{\varphi}=0$

4) Free edge: $\left\{\begin{array}{l}\bar{M}+\alpha^{2} \bar{D}(1-v) \bar{W}=0 \\ \bar{Q}-\alpha^{2}(1-v) \bar{D} \bar{\varphi}=0\end{array}\right.$

Thus, the spectral formulated task (17) and (21) the parameter $\alpha^{2}$, describes the propagation of flexural waves in planar waveguide made as a band with an arbitrary coordinate on the thickness change $x_{2}$. It is shown that the spectral parameter $\alpha^{2}$ It takes complex values (in the case of $R_{E n}(t-\tau) \neq 0$ ) If $R_{E n}(t-\tau)=0$, whereas the spectral parameter $\alpha^{2}$ It takes only real values. Transform this system (17). We have

$$
\bar{Q}^{\prime}=\bar{M}^{\prime \prime}+\bar{D}^{\prime \prime}(1-v) \alpha^{2} \bar{W}+\bar{D}^{\prime}(1-v) \alpha^{2} \bar{\varphi}
$$

From whence

$$
M^{\prime \prime}+\bar{D}^{\prime \prime}(1-v) \alpha^{2} \bar{W}-\alpha^{2} \bar{M}-\rho h w^{2} \bar{W}=0
$$

Moreover 


$$
\bar{W}-\frac{1}{\bar{D}} \bar{M}-\alpha^{2} \bar{W}=0
$$

Thus, the conversion system is of the form

$$
\left\{\begin{array}{l}
\bar{M}^{\prime \prime}-\alpha^{2} \bar{M}-\left(\rho h \omega^{2}-\bar{D}^{\prime \prime}(1-v) \alpha^{2}\right) \bar{W}=0 \\
W^{\prime \prime}-\alpha^{2} \bar{W}-\frac{1}{\bar{D}} \bar{M}=0
\end{array}\right.
$$

The boundary conditions (18)-(21) in alternating $\bar{W}, \bar{M}$ it has the form:

1) Swivel bearing: $\bar{W}=\bar{M}=0$;

2) Sliding clamp: $\bar{W}^{\prime}=\bar{M}^{\prime}-\alpha^{2} \bar{D}^{\prime}(1-v) \bar{W}=0$;

3) Anchorage: $\bar{W}=\bar{W}^{\prime}=0$

4) Free edge:

$$
\bar{M}^{\prime}+\alpha^{2} \bar{D}(1-v) \bar{W}=0
$$

$$
\bar{M}^{\prime}-\alpha^{2}(1-v)(D \bar{W})^{\prime}=0
$$

at $x_{2}=0$ or $x_{2}=+l_{2}$.

Let $\bar{M}$ and $\bar{W}$ some own functions of the system (22)-(26) may have a complex meaning. Multiply the equation system (22) to function $\widehat{\hat{M}}$ and $\widehat{\hat{W}}$, complex conjugate to $\bar{M}$ and $\bar{W}$. Identical converting the first equation, we integrate the resulting equality $x_{2}$ and composed of the following linear combination

$$
\begin{aligned}
& \int_{0}^{l_{2}} \bar{M} \bar{W}^{\prime \prime \hat{\hat{W}}} \mathrm{~d} x_{2}-\alpha^{2}(1-v) \int_{0}^{l_{2}}(\bar{D} \bar{W})^{\prime \prime} \overline{\hat{W}} \mathrm{~d} x_{2}+\alpha^{2}(1-v) \int_{0}^{l_{2}}(\bar{D} \bar{W})^{\prime \prime} \hat{\hat{W}} \mathrm{~d} x_{2} \\
& -\alpha^{2} \int_{0}^{l_{2}} \bar{M} \overline{\hat{W}} \mathrm{~d} x_{2}-\omega^{2} \int_{0}^{l_{2}} \rho h \bar{W} \hat{\hat{W}} \mathrm{~d} x_{2}-\alpha^{2}(1-v) \int_{0}^{l_{2}} \overline{D^{\prime \prime}} \overline{\bar{W}} \overline{\hat{W}} \mathrm{~d} x_{2} \\
& +\int_{0}^{l_{2}} \bar{W}^{\prime \prime} \hat{\hat{M}} \mathrm{~d} x_{2}-\alpha^{2} \int_{0}^{l_{2}} \bar{W} \hat{\hat{M}} \mathrm{~d} x_{2}-\int_{0}^{l_{2}} \frac{\bar{M} \hat{\hat{M}}}{\bar{D}} \mathrm{~d} x_{2}=0
\end{aligned}
$$

Integrating (27) by parts,

$$
\begin{aligned}
& {\left.\left[\bar{M}^{\prime}-\alpha^{2}(1-v)\left(D \bar{W}^{\prime}\right)\right] \hat{\hat{W}}\right|_{0} ^{l_{2}}-\int_{0}^{l_{2}}\left[\bar{M} \bar{W}^{\prime}+\hat{\hat{M}}^{\prime} \bar{W}^{\prime}\right] \mathrm{d} x_{2}} \\
& +\alpha^{2}(1-v) \int_{0}^{l_{2}} \bar{D} \bar{W}^{\prime} \bar{W}^{\prime} \mathrm{d} x_{2}-\alpha^{2} \int_{0}^{l_{2}}\left[\bar{M} \bar{W}^{\prime}+\hat{\hat{M}} \bar{W}\right] \mathrm{d} x_{2} \\
& +2 \alpha^{2}(1-v) \int_{0}^{l_{2}} \overline{D^{\prime \prime}} \bar{W} \hat{\hat{W}} \mathrm{~d} x_{2}-\omega^{2} \int_{0}^{l_{2}} \rho h \bar{W} \hat{\hat{W}} \mathrm{~d} x_{2}-\int_{0}^{l_{2}} \frac{\bar{M} \overline{\hat{M}}}{\bar{D}} \mathrm{~d} x_{2}+\left.\bar{W}^{\prime} \hat{\hat{M}}^{l_{2}}\right|_{0} ^{l_{2}} \\
& +2 \alpha^{2}(1-v) \int_{0}^{l_{2}} \bar{D}^{\prime} \bar{W} \overline{\hat{W}}^{\prime} \overline{\mathrm{W}} \mathrm{d} x_{2}+\alpha^{2}(1-v) \int_{0}^{l_{2}} \bar{D}^{\prime} \overline{\hat{W}}^{\prime} \overline{\hat{W}}^{\prime} \mathrm{d} x_{2}+\alpha^{2}(1-v) \int_{0}^{l_{2}} D \bar{W}^{\prime \prime} \overline{\hat{W}} \mathrm{~d} x_{2}=0
\end{aligned}
$$

or

$$
\begin{aligned}
& {\left.\left[\bar{M}^{\prime}-\alpha^{2}(1-v)(D \bar{W})^{\prime}\right] \widehat{\hat{W}}\right|_{0} ^{l_{2}}+\left.\left[\hat{\hat{M}}+\alpha^{2}(1-v) \bar{D} \widehat{\hat{W}}\right] \bar{W}^{\prime}\right|_{0} ^{l_{2}}} \\
& -\int_{0}^{l_{2}}\left(\bar{M}^{\prime} \overline{\hat{W}}^{\prime}+\overline{\hat{M}}^{\prime} \bar{W}^{\prime}\right) \mathrm{d} x_{2}-\alpha^{2} \int_{0}^{l_{2}}(\bar{W} \overline{\hat{M}}+\overline{\hat{W}} \bar{M}) \mathrm{d} x_{2}-\int_{0}^{l_{2}} \frac{\bar{M} \overline{\hat{M}}}{\bar{D}} \mathrm{~d} x_{2} \\
& -\omega^{2} \int_{0}^{l_{2}} \rho h \bar{W} \overline{\hat{W}} \mathrm{~d} x_{2}-2 \alpha^{2}(1-v) \int_{0}^{e_{2}} \overline{D^{\prime \prime}} \overline{\hat{W}} \bar{W} \mathrm{~d} x_{2}+\alpha^{2}(1-v) \int_{0}^{e_{2}} \overline{D^{\prime}}(\overline{\hat{W}} \bar{W})^{\prime} \mathrm{d} x_{2}=0 .
\end{aligned}
$$


It is easy to make sure that is the integral terms of (28) vanish at any combination of the boundary conditions (23)-(26). It should also be noted that all the functions under the integral valid at $R_{E n}(t-\tau)=0$. The expressing $\alpha^{2}(28) \mathrm{We}$ find that

$$
\alpha^{2}=\frac{\int_{0}^{l_{2}}\left(\bar{M}^{\prime} \bar{W}^{\prime}+\bar{M}^{\prime} \widehat{W}^{\prime}\right) \mathrm{d} x_{2}+\int_{0}^{l_{2}} \frac{\bar{M} \hat{\hat{M}}}{\bar{D}} \mathrm{~d} x_{2}+\omega^{2} \int_{0}^{l_{2}} \rho h \hat{\bar{W}} \widehat{\hat{W}} \mathrm{~d} x_{2}}{\int_{0}^{l_{2}}(\bar{M} \overline{\hat{W}}+\hat{\hat{M}} \bar{W}) \mathrm{d} x_{2}-2(1-v) \int_{0}^{l_{2}} \overline{D^{\prime \prime}} \overline{\hat{W}} \widehat{\hat{W}} \mathrm{~d} x_{2}-(1-v) \int_{0}^{l_{2}} \overline{D^{\prime}}(\bar{W} \overline{\hat{W}})^{\prime} \mathrm{d} x_{2}} \text {-real }
$$

number.

Thus (with $R_{E n}(t-\tau)=0$ ), It is shown that the square of the wave number for own endless strip of varying thickness is valid for any combination of boundary conditions. If $R_{E n}(t-\tau) \neq 0$, then $\alpha^{2}$ It is a complex value for any combination of boundary conditions.

\section{Adjoin Spectral Problem, Orthogonality Condition}

The resulting spectral problem (17)-(21) is not self-adjoin. Built for her adjoin problem using this Lagrange formula [9]

$$
\int_{0}^{l} L(U) \cdot V^{*} \mathrm{~d} x=\left.Z\left(U, V^{*}\right)\right|_{0} ^{l}-\int_{0}^{l} L^{*}\left(V^{*}\right) \cdot U \mathrm{~d} x,
$$

where $L$ and $L^{*}$-direct and adjoin linear differential operators; $U$ and $V^{\star}$-arbitrary decisions of relevant boundary value problems.

In our case

$$
L=\left[\begin{array}{cccc}
\frac{\partial}{\partial x_{2}} & -\alpha^{2} & -\alpha^{2} \bar{D}^{\prime}(1-v) & -\rho h \omega^{2} \\
-1 & \frac{\partial}{\partial x_{2}} & 0 & -\alpha^{2} \bar{D}^{\prime}(1-v) \\
0 & \frac{1}{\bar{D}} & \frac{\partial}{\partial x_{2}} & -\alpha^{2} \\
0 & 0 & -1 & \frac{\partial}{\partial x_{2}}
\end{array}\right]
$$

on the left-hand side of Equation (29) will be as follows

$$
\begin{aligned}
& \int_{0}^{l_{2}}\left[\bar{Q}^{\prime} \bar{Q}^{\bullet}-\alpha^{2} \bar{M} \bar{Q}^{\bullet}-\alpha^{2} \bar{D}^{\prime}(1-v) \bar{\varphi} Q^{\bullet}-\rho h \omega^{2} \bar{W} \bar{Q}^{\bullet}+\bar{M}^{\prime} \bar{M}^{\bullet}-\bar{Q} \bar{M}^{\bullet}\right. \\
& \left.+\alpha^{2} \bar{D}^{\prime}(1-v) \bar{W} \bar{M}^{\bullet}+\bar{\varphi}^{\prime} \bar{\varphi}^{\bullet}-\frac{1}{\bar{D}} \bar{M} \bar{\varphi}^{\bullet}-\alpha^{2} \bar{W} \bar{\varphi}^{\bullet}+\bar{W}^{\prime} \bar{W}^{\bullet}-\bar{\varphi} \overline{W^{\bullet}}\right] \mathrm{d} x_{2}=0
\end{aligned}
$$

or, integrating parts

$$
\begin{aligned}
& {\left.\left[\bar{Q} \bar{Q}^{\bullet}+\bar{M} \bar{M}^{\bullet}+\bar{\varphi} \bar{\varphi}^{\bullet}+\bar{W} \bar{W}^{\bullet}\right]\right|_{0} ^{l_{2}}-\int_{0}^{l_{2}}\left[\left(\bar{Q}^{\bullet \prime}+\bar{M}^{\bullet}\right) \bar{Q}\right.} \\
& +\left(\bar{M}^{\bullet \prime}+\alpha^{2} \bar{Q}^{\bullet}+\frac{1}{\bar{D}} \bar{\varphi}^{\bullet}\right) \bar{M}+\left(\bar{\varphi}^{\bullet \prime}+\bar{W}^{\bullet}+\alpha^{2} \bar{D}^{\prime}(1-v) \bar{Q}^{\bullet}\right) \bar{\varphi} \\
& \left.+\left(\bar{W}^{\bullet \prime}+\alpha^{2} \bar{\varphi}^{\bullet}-\alpha^{2} \bar{D}^{\prime}(1-v) \bar{M}^{\bullet}+\rho h \omega^{2} \bar{Q}^{\bullet}\right) \bar{W}\right] \mathrm{d} x_{2}=0
\end{aligned}
$$


Thus the conjugate (30)-(32), the system has the form

$$
\left\{\begin{array}{l}
\bar{Q}^{\bullet \prime}+\bar{M}^{\bullet}=0 \\
\bar{M}^{\bullet \prime}+\alpha^{2} \bar{Q}^{\bullet}+\frac{1}{D} \bar{\varphi}^{\bullet}=0 \\
\bar{\phi}^{\bullet \prime}+\bar{W}^{\bullet}+\alpha^{2} \bar{D}^{\prime}(1-v) \bar{Q}^{\bullet}=0 \\
\bar{W}^{\bullet \prime}-\alpha^{2} \bar{D}^{\prime}(1-v) \bar{M}^{\bullet}+\alpha^{2} \bar{\varphi}^{\bullet}+\rho h \omega^{2} \bar{Q}^{\bullet}=0
\end{array}\right.
$$

Moreover, we get the conjugate boundary conditions of equality to zero is integral members $\left.Z\left(U, V^{*}\right)\right|_{0} ^{l_{2}}$ expression in (32):

1) Swivel bearing: $\bar{\varphi}^{\bullet}=\bar{Q}^{\bullet}=0, x_{2}=0, l_{2}$

2) Sliding clamp: $\bar{W}^{\bullet}=\bar{M}^{\bullet}=0, \quad x_{2}=0, l_{2}$

3) Anchorage: $\bar{M}^{\bullet}=\bar{Q}^{\bullet}=0, x_{2}=0, l_{2}$

4) Free edge: $\left\{\begin{array}{l}\bar{\varphi}^{\bullet}+\alpha^{2} \bar{D}(1-v) \bar{Q}^{\bullet}=0, \\ \bar{W}^{\bullet}-\alpha^{2} \bar{D}(1-v) \bar{M}^{\bullet}=0, \quad x_{2}=0, l_{2}\end{array}\right.$

For conditions biorthogonality solutions once again use the Lagrange formula (29) in the form

$$
\int_{0}^{l}\left[L(U) V^{\bullet}+L^{\bullet}\left(V^{\bullet}\right) U\right] \mathrm{d} x=\left.Z\left(U, V^{\bullet}\right)\right|_{0} ^{l_{2}},
$$

that leads to the consideration of the following integral

$$
\begin{aligned}
& \int_{0}^{l_{2}}\left[\bar{Q}_{i}^{\prime} \bar{Q}_{j}^{\bullet}-\alpha_{i}^{2} \bar{M}_{i} \bar{Q}_{j}^{\bullet}-\alpha_{i}^{2} \bar{D}^{\prime}(1-v) \bar{\varphi}_{i} \bar{Q}_{j}^{\bullet}-\rho h \omega^{2} \bar{W}_{i} \bar{Q}_{j}^{\bullet}+\bar{M}_{i}^{\prime} \bar{M}_{j}^{\bullet}\right. \\
& -\bar{Q}_{i} \bar{M}_{j}^{\bullet}+\alpha_{i}^{2} \bar{D}^{\prime}(1-v) \bar{W}_{i} \bar{M}_{j}^{\bullet}+\bar{\varphi}_{i}^{\prime} \bar{\varphi}_{j}^{\bullet}-\frac{1}{\bar{D}} \bar{M}_{i} \bar{\varphi}_{j}^{\bullet}-\alpha_{i}^{2} \bar{W}_{i} \bar{\varphi}_{j}^{\bullet} \\
& +\bar{W}_{i}^{\prime} \bar{W}_{j}^{\bullet}-\bar{\varphi}_{i} \bar{W}_{j}^{\bullet}+\bar{Q}_{j}^{\bullet \prime} \bar{Q}_{i}+\bar{M}_{j}^{\bullet} \bar{Q}_{i}+\bar{M}_{j}^{\bullet \prime} \bar{M}_{i}+\alpha_{j}^{2} \bar{M}_{i} \bar{Q}_{j}^{\bullet} \\
& +\frac{1}{\bar{D}} \bar{M}_{i} \bar{\varphi}_{j}^{\bullet}+\bar{\varphi}_{i} \bar{\varphi}_{j}^{\bullet \prime}+\bar{W}_{j}^{\bullet} \bar{\varphi}_{i}+\alpha_{j}^{2} D^{\prime}(1-v) Q_{j}^{\bullet} \bar{\varphi}_{i}+\bar{W}_{i} \bar{W}_{j}^{\bullet \prime} \\
& \left.+\alpha_{j}^{2} \bar{W}_{i} \bar{\varphi}_{j}^{\bullet}-\alpha_{j}^{2} \bar{D}^{\prime}(1-v) \bar{W}_{i} \bar{M}_{j}^{\bullet}+\rho h \omega^{2} \bar{Q}_{j}^{\bullet} \bar{W}_{i}\right] \mathrm{d} x_{2}=0,
\end{aligned}
$$

where $\left(\bar{Q}_{i}, \bar{M}_{i}, \bar{\varphi}_{i}, \bar{W}_{i}\right)^{\mathrm{T}}$-own form, corresponding to the Eigen value $\alpha_{\mathrm{i}}$ original spectral problem; $\left(\bar{Q}_{j}^{\bullet}, \bar{M}_{j}^{\bullet}, \bar{\varphi}_{j}^{\bullet}, \bar{W}_{j}^{\bullet}\right)^{\mathrm{T}}$-own form, corresponding to the Eigen value $\alpha_{\mathrm{j}}$ adjoin.

Integrating (38) by parts

$$
\begin{aligned}
& \left(\alpha_{i}^{2}-\alpha_{j}^{2}\right)\left[\int_{0}^{l_{2}}\left[-\bar{M}_{i} \bar{Q}_{j}^{\bullet}-\bar{D}^{\prime}(1-v) \bar{Q}_{j}^{\bullet} \bar{\varphi}_{i}+\bar{D}^{\prime}(1-v) \bar{W}_{i} \bar{M}_{j}^{\bullet}-\bar{W}_{i} \bar{\varphi}_{j}^{\cdot}\right] \mathrm{d} x_{2}\right. \\
& \left.+\left.\left[\bar{D}(1-v) \bar{Q}_{j}^{\bullet} \bar{\varphi}_{i}-\bar{D}(1-v) \bar{W}_{i} \bar{M}_{j}^{\bullet}\right]\right|_{0} ^{l_{2}}\right]=0,
\end{aligned}
$$

where to $i \neq j$ we have the condition biorthogonality forms:

$$
\begin{aligned}
& \int_{0}^{l_{2}}\left[\left(\bar{M}_{i}+\bar{D}^{\prime}(1-v) \bar{\varphi}_{i}\right) \bar{Q}_{j}^{\bullet}+\bar{W}_{i}\left(\bar{\varphi}_{j}^{\bullet}-\bar{D}^{\prime}(1-v) \bar{M}_{j}^{\bullet}\right)\right] \mathrm{d} x_{2} \\
& +\left.\bar{D}(1-v)\left[\bar{W}_{i} \bar{M}_{j}^{\bullet}-\bar{Q}_{j}^{\bullet} \bar{\varphi}_{i}\right]\right|_{0} ^{l_{2}}=\delta_{i j}
\end{aligned}
$$


The expression $\bar{W}_{i} \bar{M}_{j}^{\bullet}-\bar{Q}_{j}^{\bullet} \bar{\varphi}_{i} \quad$ zero, if the border is set to any of the conditions (18)-(21) in addition to the conditions of the free edge.

\section{Fixed Problem for a Semi-Infinite Strip of Variable Thickness}

Consider a semi-infinite axial $x_{1}$ lane variable section, wherein at the end $\left(x_{1}=0\right)$ harmonic set time exposure of one of two types of:

$$
W=f_{W}\left(x_{2}\right) \mathrm{e}^{i \omega t}, M_{11}=f_{W}\left(x_{2}\right) \mathrm{e}^{i \omega t}, x_{l}=0
$$

or

$$
\varphi_{1}=f_{\varphi}\left(x_{2}\right) \mathrm{e}^{i \omega t}, \quad Q_{1}=f_{Q}\left(x_{2}\right) \mathrm{e}^{i \omega t}, \quad x_{1}=0
$$

where

$$
\varphi_{1}=\frac{\partial W}{\partial x_{1}}, \quad Q_{1}=\bar{D}\left[\frac{\partial^{3} W}{\partial x_{1}^{3}}+2(1-v) \frac{\partial^{3} W}{\partial x_{1} \partial x_{2}^{2}}\right]
$$

Transform the boundary conditions (41) so that they contain only selected our variables $W, \varphi, M$ and $Q$

$$
\begin{gathered}
W=f_{w}\left(x_{2}\right) \mathrm{e}^{i \omega t}, \quad \bar{D}\left(\frac{\partial^{2} W}{\partial x_{1}^{2}}+v \frac{\partial^{2} W}{\partial x_{2}^{2}}\right)=f_{M}\left(x_{2}\right) \mathrm{e}^{i \omega t}, x_{1}=0, \\
\frac{\partial W}{\partial x_{1}}=f_{\varphi}\left(x_{2}\right) \mathrm{e}^{i \omega t}, \bar{D}\left(\frac{\partial^{3} W}{\partial x_{1}^{3}}+2(1-v) \frac{\partial^{3} W}{\partial x_{1} \partial x_{2}^{2}}\right)=f_{Q}\left(x_{2}\right) \mathrm{e}^{i \omega t}, x_{1}=0,
\end{gathered}
$$

or

$$
\begin{gathered}
W=f_{w}\left(x_{2}\right) \mathrm{e}^{i \omega t}, \quad \bar{D}\left(\frac{\partial^{2} W}{\partial x_{1}^{2}}+v \frac{\partial^{2} W}{\partial x_{2}^{2}}\right)-\bar{D}(1-v) \frac{\partial^{2} W}{\partial x_{2}^{2}}=f_{M}\left(x_{2}\right) \mathrm{e}^{i \omega t}, x_{1}=0 \\
\frac{\partial W}{\partial x_{1}}=f_{\varphi}\left(x_{2}\right) \mathrm{e}^{i \omega t} \\
\frac{\partial}{\partial x_{1}}\left[\bar{D}\left(\frac{\partial^{2} W}{\partial x_{1}^{2}}+\frac{\partial^{2} W}{\partial x_{2}^{2}}\right)\right]+\bar{D}(1-v) \frac{\partial^{2}}{\partial x_{2}^{2}}\left(\frac{\partial W}{\partial x_{1}}\right)=f_{Q}\left(x_{2}\right) \mathrm{e}^{i \omega t}, x_{1}=0,
\end{gathered}
$$

Of finally

$$
\begin{gathered}
W=f_{w}\left(x_{2}\right) \mathrm{e}^{i \omega t}, M=\left[f_{M}\left(x_{2}\right)+\bar{D}(1-v) f_{w}^{\prime \prime}\left(x_{2}\right)\right] \mathrm{e}^{i \omega t}, x_{1}=0 \\
\frac{\partial W}{\partial x_{1}}=f_{\varphi}\left(x_{2}\right) \mathrm{e}^{i \omega t}, \\
\frac{\partial M}{\partial x_{1}}=\left[f_{Q}\left(x_{2}\right)-\bar{D}(1-v) f_{\varphi}^{\prime \prime}\left(x_{2}\right)\right] \mathrm{e}^{i \omega t}, x_{1}=0 .
\end{gathered}
$$

Assume that the desired solution of the no stationary problem can be expanded in a series in Eigen functions of the solution of the spectral problem. In the case of constant thickness it is evident, and in general, the question remains open.

The solution of the stationary problem (17)-(21) (41)-(42) will seek a 


$$
\left(\begin{array}{c}
W \\
\varphi \\
M \\
Q
\end{array}\right)=\sum_{k=1}^{N} a_{k}\left(\begin{array}{c}
\bar{W}_{k}\left(x_{2}\right) \\
\bar{\varphi}_{k}\left(x_{2}\right) \\
\bar{M}_{k}\left(x_{2}\right) \\
\bar{Q}_{k}\left(x_{2}\right)
\end{array}\right) \mathrm{e}^{-i\left(\alpha_{k} x_{1}-\omega t\right)} .
$$

where $\bar{W}_{k}, \bar{\varphi}_{k}, \bar{\theta}_{k}, \bar{M}_{k}$-biorthonormal own forms of the spectral problem (17) (21).

The representation (46) gives us the solution to the problem of non-stationary wave in the far field, i.e., where it has faded not propagating modes. The number of propagating modes used $\mathrm{N}$ course for each specific frequency $\omega$, since the $\mathrm{cu}-$ toff frequency is greater than the other $\omega$.

Consider two cases of excitation of stationary waves in the band:

1) $f_{w}=0$-antisymmetric relative $x_{1}$;

2) $f_{\varphi}=0$-symmetric.

In the case of antisymmetric excitation, substituting (46) into (44) and expressing $f_{M}\left(X_{2}\right)$, obtain

$$
f_{M}\left(x_{2}\right)=\sum_{k=1}^{N} \alpha_{k} \bar{M}_{k}\left(x_{2}\right) \text {. }
$$

Value biorthogonality (40) gives expression to determine the unknown coefficients $a_{k}=\int_{0}^{e_{2}} f_{M}\left(x_{2}\right) \bar{Q}_{k}^{\bullet}\left(x_{2}\right) \mathrm{d} x_{2}$.

In the case of a symmetrical excitation $f_{Q}\left(x_{2}\right)$ We obtain rearranging (46) to (45) in the following form

$$
f_{Q}\left(x_{2}\right)=\sum_{k=1}^{N}\left(-i \alpha_{k} a_{k} \bar{M}_{k}\left(x_{2}\right)\right)
$$

Biorthogonality ratio (30) gives

$$
a_{k}=\frac{i}{\alpha_{k}} \int_{0}^{l_{2}} f_{Q}\left(x_{2}\right) \bar{Q}_{k}^{\cdot} \mathrm{d} x_{2}
$$

\section{Testing Software System and Study the Properties of Propagation of Flexural Waves in a Band of Variable Thickness}

Testing program was carried out on the task of distributing the flexural waves in a plate of constant thickness. Consider the floor plate of constant thickness infinitely satisfying Kirchhoff-Love hypotheses, with supported long edges (Figure 1).

At end face $x_{1}=0$ specified:

$$
w=f_{1}\left(x_{2}\right) \mathrm{e}^{i w t}, M_{11}=f_{2}\left(x_{2}\right) \mathrm{e}^{i w t}
$$

Spread along the axis $X_{1}$ flexural wave is described by the differential control system (13) 


$$
\left\{\begin{array}{l}
Q^{\prime}+\frac{\partial^{2} M}{\partial x_{1}^{2}}+\rho h w^{\prime \prime}=0\left(w^{\prime \prime}=\partial^{2} w / \partial t^{2}\right), \\
M^{\prime}-\theta=0, \\
\varphi^{\prime}-\frac{1}{\bar{D}} M+\frac{\partial^{2} w}{\partial x_{1}^{2}}=0 \\
w^{\prime}-\varphi=0, h=h_{0}
\end{array}\right.
$$

with boundary conditions of the form (15)

$$
w=0, M-\bar{D}(1-v) \frac{\partial^{2} w}{\partial x_{1}^{2}}=0, x_{2}=0, \pi
$$

Introducing the desired motion vector in the form of

$$
\left(\begin{array}{c}
Q \\
M \\
\varphi \\
w
\end{array}\right)=\left(\begin{array}{c}
\bar{Q} \\
\bar{M} \\
\bar{\varphi} \\
\bar{W}
\end{array}\right) \mathrm{e}^{-i\left(a_{k} x_{1}-\omega t\right)}
$$

Go to the spectral problem

$$
\left\{\begin{array}{l}
\bar{Q}^{\prime}-\alpha^{2} \bar{M}-\omega^{2} \rho h \bar{W}=0, \\
\bar{M}^{\prime}-\bar{Q}=0, \\
\bar{\varphi}^{\prime}-\frac{1}{\bar{D}} \bar{M}-\alpha^{2} \bar{W}=0, \\
\bar{W}^{\prime}-\bar{\varphi}=0
\end{array}\right.
$$

with the boundary conditions

$$
W=0, \bar{M}+\bar{D}(1-v) \alpha^{2} \bar{W}=0, \quad x_{2}=0, \pi
$$

or

$$
\bar{W}=0, \bar{M}=0, x_{2}=0, \pi .
$$

Rewrite the system (54) as follows

$$
\left\{\begin{array}{l}
\bar{W}^{\prime \prime}-\frac{1}{\bar{D}} \bar{M}-\alpha^{2} \bar{W}=0, \\
\bar{M}^{\prime \prime}-\alpha^{2} \bar{M}-\omega^{2} \rho h \bar{W}=0,
\end{array}\right.
$$

and $\bar{W}=0, \bar{M}=0, x_{2}=0, \pi$.

We seek the solution of (56) in the form

$$
\begin{aligned}
& \bar{W}=a_{w} \sin n x_{2}, \\
& M=a_{M} \sin n x_{2},(n=1,2, \cdots)
\end{aligned}
$$

satisfying the boundary conditions (55).

We obtain an algebraic homogeneous system

$$
\begin{aligned}
& -n^{2} a_{W}-\alpha^{2} a_{W}-\frac{1}{\bar{D}} a_{M}=0 ; \\
& -n^{2} a_{M}-\alpha^{2} a_{M}-\omega^{2} \rho h a_{w}=0 .
\end{aligned}
$$

For the existence of a nontrivial solution, which is necessary to require the vanishing of its determinant 


$$
\operatorname{det}\left|\begin{array}{cc}
n^{2}+\alpha^{2} & \frac{1}{\bar{D}} \\
\omega^{2} \rho h & n^{2}+\alpha^{2}
\end{array}\right|=0,
$$

or

$$
\alpha_{1,2}^{2}+n^{2}= \pm \omega \sqrt{\frac{\rho h}{\bar{D}_{k}}}
$$

where, when $R_{E n}(t-\tau)=0$

$$
\alpha_{1,2}^{2}=-n^{2} \pm \omega \sqrt{\frac{\rho h}{D}} .
$$

Ownership of constant thickness strip bending vibrations are of the form

$$
\begin{aligned}
& \bar{W}_{n}^{1,2}=\sin n x_{2} ; \\
& M_{n}^{1,2}= \pm \omega \sqrt{\rho h \bar{D}} \sin n x_{2} ; \\
& \varphi_{n}^{1,2}=n \cos n x_{2} ; \\
& Q_{n}^{1,2}=\mp \omega \sqrt{\rho h \bar{D}} \cos n x_{2} .
\end{aligned}
$$

We construct the solution of the problem adjoin to (54)-(55)

$$
\left\{\begin{array}{l}
\bar{W}^{\bullet \prime}+\alpha^{2} \bar{\varphi}^{\bullet}+\rho h \omega^{2} \bar{Q}^{*}=0 \\
\bar{\varphi}^{\bullet \prime}+W^{\bullet}=0 \\
\bar{M}^{* \prime}+\frac{1}{\bar{D}} \bar{\varphi}^{\bullet}+\alpha^{2} \bar{Q}^{\bullet}=0 \\
\bar{Q}^{\bullet \prime}+\bar{M}^{\bullet}=0
\end{array}\right.
$$

and

$$
\bar{\varphi}^{*}=0, \bar{Q}^{*}=0, x_{2}=0, \pi .
$$

Transforming (61)-(62) we obtain the following system of first order differential equations

$$
\begin{aligned}
& \left\{\begin{array}{l}
\bar{\varphi}^{\bullet \prime}-\alpha^{2} \bar{\varphi}^{\bullet}-\rho h \omega^{2} \bar{Q}^{\bullet}=0 \\
\bar{Q}^{\bullet \prime}-\alpha^{2} \bar{Q}^{\bullet}-\frac{1}{\bar{D}} \bar{\varphi}^{\bullet}=0,
\end{array}\right. \\
& x_{2}=0, \pi ; \bar{\varphi}^{\bullet}=0, \bar{Q}^{\bullet}=0 .
\end{aligned}
$$

The solution of (63) in the form

$$
\bar{\varphi}^{\bullet}=a_{\varphi}^{\bullet} \sin n x_{2}, \quad \bar{Q}^{\bullet}=a_{Q}^{\bullet} \sin n x_{2}
$$

From whence $\alpha_{1,2}^{2}$ It has the same form (59a), own forms of vibrations are of the form:

$$
\begin{aligned}
& \bar{\varphi}_{n}^{\bullet 1,2}= \pm \omega \sqrt{p h \bar{D}} \sin n x_{2}, \\
& \bar{Q}_{n}^{\bullet 1,2}=\sin n x_{2}, \\
& \bar{W}_{n}^{\bullet 1,2}= \pm n \omega \sqrt{p h \bar{D}} \cos n x_{2} \\
& \bar{M}_{n}^{\bullet 1,2}=n \cos n x_{2} .
\end{aligned}
$$


For biorthogonality conditions direct solutions and the adjoin problem is necessary to consider the following equation

$$
\begin{aligned}
& \int_{0}^{\pi}\left[\bar{Q}_{i}^{\prime} \bar{Q}_{j}^{\bullet}-\alpha_{i}^{2} \bar{M}_{i} \bar{Q}_{j}^{\bullet}-\omega^{2} \rho h \bar{W}_{i} \bar{Q}_{j}^{\bullet}+\bar{Q}_{j}^{\bullet \prime} \bar{Q}_{i}+\bar{M}_{j}^{\bullet} \bar{Q}_{i}+\bar{M}_{i}^{\prime} \bar{M}_{j}^{\bullet}-\bar{Q}_{i} \bar{M}_{j}^{\bullet}\right. \\
& +\bar{M}_{j}^{\cdot \prime} \bar{M}_{i}+\frac{1}{\bar{D}} \bar{\varphi}_{j}^{\cdot} \bar{M}_{i}+\alpha_{j}^{2} \bar{Q}_{j}^{\cdot} \bar{M}_{i}+\bar{\varphi}_{i}^{\prime} \bar{\varphi}_{j}^{\cdot}-\frac{1}{\bar{D}} \bar{M}_{i} \bar{\varphi}_{j}^{\cdot}-\alpha_{i}^{2} \bar{W}_{i} \bar{\varphi}_{j}^{\cdot}+\bar{\varphi}_{j}^{\circ} \bar{\varphi}_{i} \\
& \left.+\bar{W}_{j}^{\bullet} \bar{\varphi}_{i}+\bar{W}_{i}^{\prime} \bar{W}_{j}^{\bullet}-\bar{\varphi}_{i} \bar{W}_{j}^{\bullet}+\bar{W}_{j}^{\bullet} \bar{W}_{i}+\alpha^{2} \bar{\varphi}_{j}^{\bullet} \bar{W}_{i}+\rho h \omega^{2} \bar{Q}_{j}^{\bullet} \bar{W}_{i}\right] \mathrm{d} x_{2}=\delta_{i j}
\end{aligned}
$$

where $\bar{Q}_{i}, \bar{M}_{i}, \bar{\varphi}_{i}, \bar{W}_{i}$-own form for the direct problem, the corresponding Eigen values $\alpha_{i}$, and $Q_{j}^{\cdot}, \bar{M}_{j}^{\bullet}, \bar{\varphi}_{j}^{\cdot}, \bar{W}_{j}^{\bullet}$-own form of the dual problem, the corresponding Eigen value $\alpha_{j}$ Integrating by parts in (66), using the boundary conditions (55) and (62) we obtain the desired condition:

$$
\int_{0}^{\pi}\left[\bar{M}_{i} \bar{Q}_{j}^{\cdot}+\bar{W}_{i} \bar{\varphi}_{j}^{\cdot}\right] \mathrm{d} x_{2}=\delta_{i j}
$$

We now verify biorthogonality received their own forms (60) and (65) using the condition biorthogonality (67)

$$
\begin{aligned}
& \int_{0}^{\pi}\left[\sqrt{\rho h \bar{D}} \sin \left(i x_{2}\right) \cdot \sin \left(j x_{2}\right)+\sqrt{\rho h \bar{D}} \sin \left(i x_{2}\right) \sin \left(j x_{2}\right)\right] \mathrm{d} x_{2} \\
& =2 \sqrt{\rho h \bar{D}} \int_{0}^{\pi} \sin \left(i x_{2}\right) \sin \left(j x_{2}\right) \mathrm{d} x_{2}=\pi \sqrt{\rho h \bar{D}} \delta_{i j}
\end{aligned}
$$

The normalized adjoin eigenvector on $\pi \sqrt{\rho h \bar{D}}$, we have a system of eigenvectors satisfying the condition (67).

We now obtain the solution of the problem of the distribution of the stationary wave in the semi-infinite strip of constant thickness. Suppose that at the border $x_{1}=0$ set the following stationary disturbance:

$$
\begin{aligned}
& w=\bar{W} \mathrm{e}^{i \omega t}=b_{W} \sin \left(n x_{2}\right) \mathrm{e}^{\mathrm{i} \omega t}, \\
& M=\bar{M} \mathrm{e}^{i \omega t}=b_{M} \sin \left(n x_{2}\right) \mathrm{e}^{i \omega t}, x_{1}=0
\end{aligned}
$$

We seek a solution of a problem

$$
w\left(x_{1}, x_{2}, t\right)=\sum_{k=1}^{\infty} a_{k} W_{k}, M\left(x_{1}, x_{2}, t\right)=\sum_{k=1}^{\infty} a_{k} M_{k}
$$

where

$$
W_{k}=\bar{W}\left(x_{21}\right) \mathrm{e}^{-i\left(a_{k} x_{l}-\omega t\right)}, M_{k}=\bar{M}\left(x_{2}\right) \mathrm{e}^{-i\left(a_{k} x_{l}-\omega t\right)},
$$

a $\bar{W}_{k} u \bar{M}_{k}$-own form (60), corresponding to $\alpha_{k}$

It is evident from the band at the end face at $x_{1}=0$ decision (69) must satisfy the boundary conditions (68)

$$
\begin{aligned}
& b_{W} \sin \left(n x_{2}\right) \mathrm{e}^{i \omega t}=\sum_{k=1}^{\infty} a_{k} \bar{W}_{k}\left(x_{2}\right) \mathrm{e}^{i \omega t}, \\
& b_{M} \sin \left(n x_{2}\right) \mathrm{e}^{i \omega t}=\sum_{k=1}^{\infty} a_{k} \bar{M}_{k}\left(x_{2}\right) \mathrm{e}^{i \omega t},
\end{aligned}
$$


or go to the amplitude values

$$
\begin{aligned}
& b_{w} \sin \left(n x_{2}\right) \mathrm{e}^{i \omega t}=\sum_{k=1}^{\infty} a_{k} \bar{W}_{k}, \\
& b_{M} \sin \left(n x_{2}\right) \mathrm{e}^{i \omega t}=\sum_{k=1}^{\infty} a_{k} \bar{M}_{k},
\end{aligned}
$$

Consider the following integral

$$
\begin{aligned}
\int_{0}^{\pi}\left[\bar{M} \bar{Q}_{j}^{\bullet}+\bar{W} \bar{\varphi}_{j}^{\bullet}\right] \mathrm{d} x_{2} & =\int_{0}^{\pi}\left[\sum_{k=1}^{\infty} a_{k} \bar{M}_{k} \bar{Q}_{j}^{\bullet}+\sum_{k=1}^{\infty} a_{k} \bar{W}_{k} \bar{\varphi}_{j}^{\bullet}\right] \mathrm{d} x_{2} \\
& =\sum_{k=1}^{\infty} a_{k} \int_{0}^{\pi}\left[\bar{M}_{k} \bar{Q}_{j}^{\bullet}+\bar{W}_{k} \bar{\varphi}_{j}^{\bullet}\right] \mathrm{d} x_{2}=a_{j} .
\end{aligned}
$$

On the other hand on the edge $x_{1}=0$ the same integral as follows

$$
\int_{0}^{\pi}\left[b_{M} \sin \left(n x_{2}\right) \bar{Q}_{j}^{\bullet}+b_{W} \sin \left(n x_{2}\right) \bar{\varphi}_{j}^{\bullet}\right] \mathrm{d} x_{2}
$$

Substituting in (72) from the normalized own form (65) we obtain

$$
\begin{aligned}
& \int_{0}^{\pi}\left[b_{M} \sin \left(n x_{2}\right) \frac{1}{\pi \sqrt{\rho h \bar{D}}} \sin \left(j x_{2}\right) \pm b_{w} \sin \left(n x_{2}\right) \frac{\omega}{\pi} \sin \left(j x_{2}\right)\right] \mathrm{d} x_{2} \\
& =\left[\frac{b_{M}}{\pi \sqrt{\rho h \bar{D}}} \pm \frac{b_{w} \omega}{\pi}\right] \cdot \int_{0}^{\pi} \sin \left(n x_{2}\right) \cdot \sin \left(j x_{2}\right) \mathrm{d} x_{2}
\end{aligned}
$$

From a comparison of the formulas (71) and (73) it is clear that under such boundary conditions is excited only " $n$ "-Single private form:

$$
a_{j}^{ \pm}=\delta_{n j}\left[\frac{b_{M}}{2 \sqrt{\rho h \bar{D}}} \pm \frac{b_{w} \omega}{2}\right]
$$

Thus, the solution of the no stationary problem for a half-strip of constant thickness has the form

$$
\begin{aligned}
& \bar{W}=\left(a_{n}^{+} \bar{W}_{n}^{+}+a_{n}^{-} \bar{W}_{n}^{-}\right) \mathrm{e}^{-i\left(\alpha x_{l}-\omega t\right)} \\
& \bar{M}=\left(a_{n}^{-} \bar{M}_{n}^{+}+a_{n}^{-} \bar{M}_{n}^{-}\right) \mathrm{e}^{-i\left(\alpha x_{l}-\omega t\right)}
\end{aligned}
$$

where

$\bar{W}_{n}^{ \pm}= \pm \sin n x_{2}, \quad \bar{M}_{n}^{ \pm}= \pm \omega \sqrt{\rho h \bar{D}} \sin n x_{2}$ a $a_{n}^{ \pm}$-determined from the ratio (74).

Now suppose that the steady influence on the border of semi-infinite strip $x_{1}=0$ it has the form

$$
w=f_{w}\left(x_{2}\right) \mathrm{e}^{i \omega t}, \quad M=f_{M}\left(x_{2}\right) \mathrm{e}^{i \omega t}
$$

Let us expand the function $f_{w}$ and $f_{M}$ Fourier series of sinus in the interval $[0, \pi]$

$$
f_{w}\left(x_{2}\right)=\sum_{k=1}^{\infty} B_{w}^{k} \sin \left(k x_{2}\right), \quad f_{M}\left(x_{2}\right)=\sum_{k=1}^{\infty} b_{M}^{k} \sin \left(k x_{2}\right)
$$

Using the results of the previous problem, we find that the solution can be represented as a Fourier series: 


$$
W=\sum_{k=1}^{\infty}\left(a_{k}^{+} W_{k}^{+}+a_{k}^{-} W_{k}^{-}\right) \mathrm{e}^{-i\left(\alpha x_{l}-\omega t\right)}, M=\sum_{k=1}^{\infty}\left(a_{k}^{+} M_{k}^{+}+a_{k}^{-} M_{k}^{-}\right) \mathrm{e}^{-i\left(\alpha x_{l}-\omega t\right)}
$$

where $a_{k}^{ \pm}=\frac{b_{M}^{k}}{2 \sqrt{\rho h \bar{D}}} \pm \frac{b_{w}^{k} \omega}{2}$ a $\bar{W}_{k}^{ \pm}, \bar{M}_{k}^{ \pm}$determined from the ratio (60).

\section{Numerical Results and Analysis}

The numerical solution of spectral problems carried out by computer software system based on the method of orthogonal shooting S. K. Godunov [10] combined with the method of Muller. The results obtained in testing with the same software package analytically up to 4 - 5 mark frequency range from 0.01 to 100 . Hereinafter, the entire analysis is conducted in dimensionless variables, in which the density of the material $\rho$, half the width of the waveguide $l_{2}$ and E modulus taken to be unity, and the parameters of relaxation kernel $A=0,048 ; \beta=0,05 ; \alpha=0,1$.

The calculation results are obtained when $A=0$. Figure 2 shows the spectral curves of the lower modes of oscillation of constant thickness plate, the corresponding $n=0,1,2,3,4,5$ for Poisson's ratio $v=0.25$. Analysis of the data shows that the range of applicability of the theory of Kirchhoff-Love to a plate of constant thickness is limited by the low frequency range. For example, for the first mode $(h=0)$, the range of application of the theory $0 \leq \omega \leq 3$ because of the unlimited growth of the phase propagation velocity with increasing frequency, for high frequencies $C_{f} C_{s} \sim \sqrt{\omega}$.

At high frequencies, where the wavelength is comparable or less than the fashion of strip thickness, there is, as is well known, localized in the faces of the Rayleigh wave band at a speed slower speed $C_{s}$, however, as is obvious, this formulation of the problem, in principle, does not allow to obtain this result. However, it should be noted that in the application of the theory of Kirchhoff-Love platinum constant thickness is obtained the correct conclusion about the growth

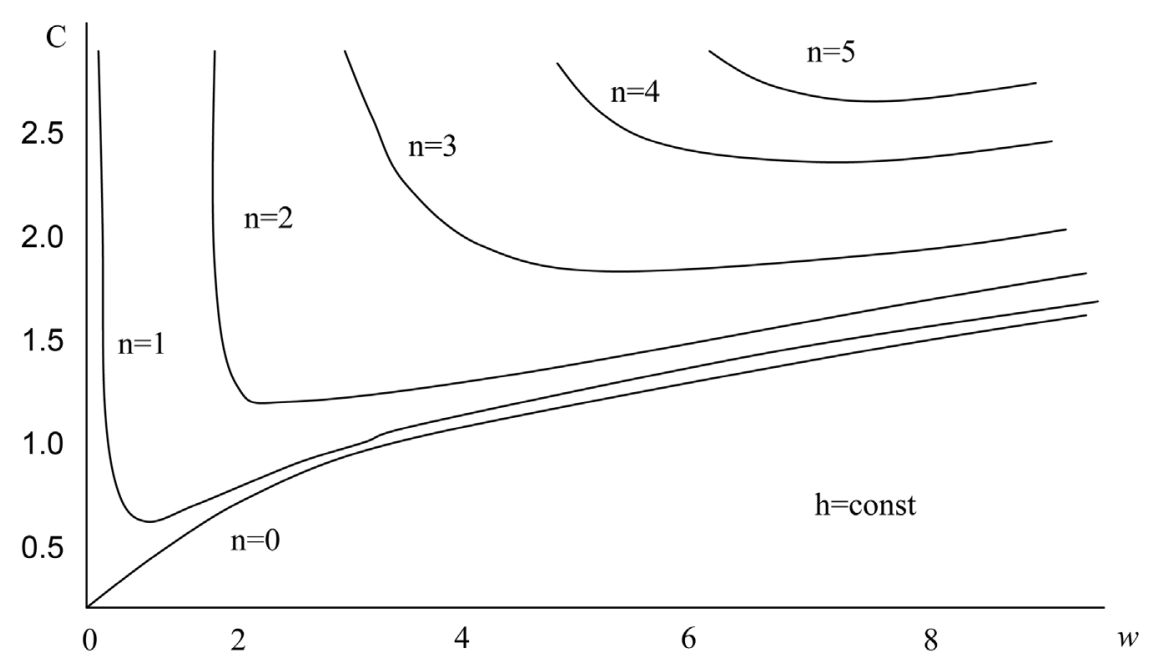

Figure 2. The dependence of the phase velocity on frequency. 
of the number of propagating modes with increasing frequency that is well seen from the spectral curves of Figure 2 and Figure 3, which shows the dependence of the wave number $\alpha$ the frequency for the same modes of waves.

Figure 4 shows the obtained numerical form for the above modes of oscillations coincided with the same accuracy ( $4-5$ decimal places) in the division of bandwidth by 90 equal segments.

Figure 5 illustrates the solution of the stationary problem: the amplitude of the excited oscillation modes linearly depends on the frequency $\omega$.

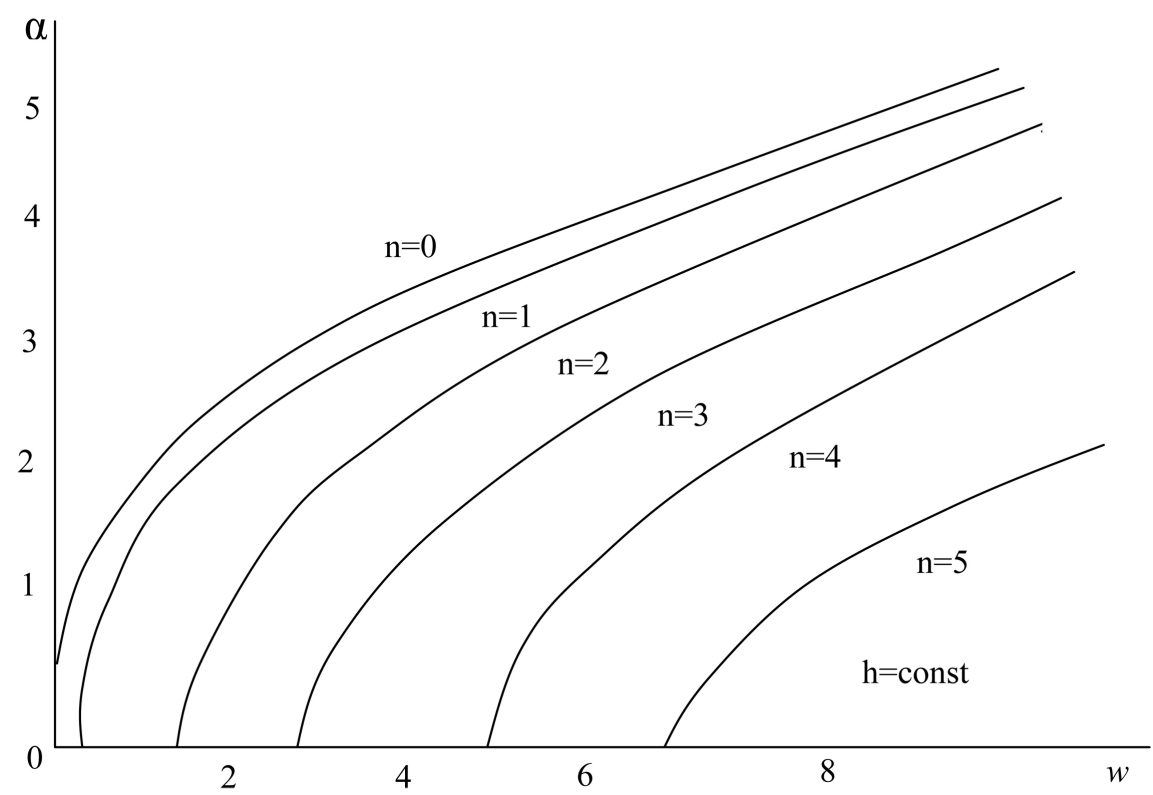

Figure 3. The dependence of the frequency of the wave.

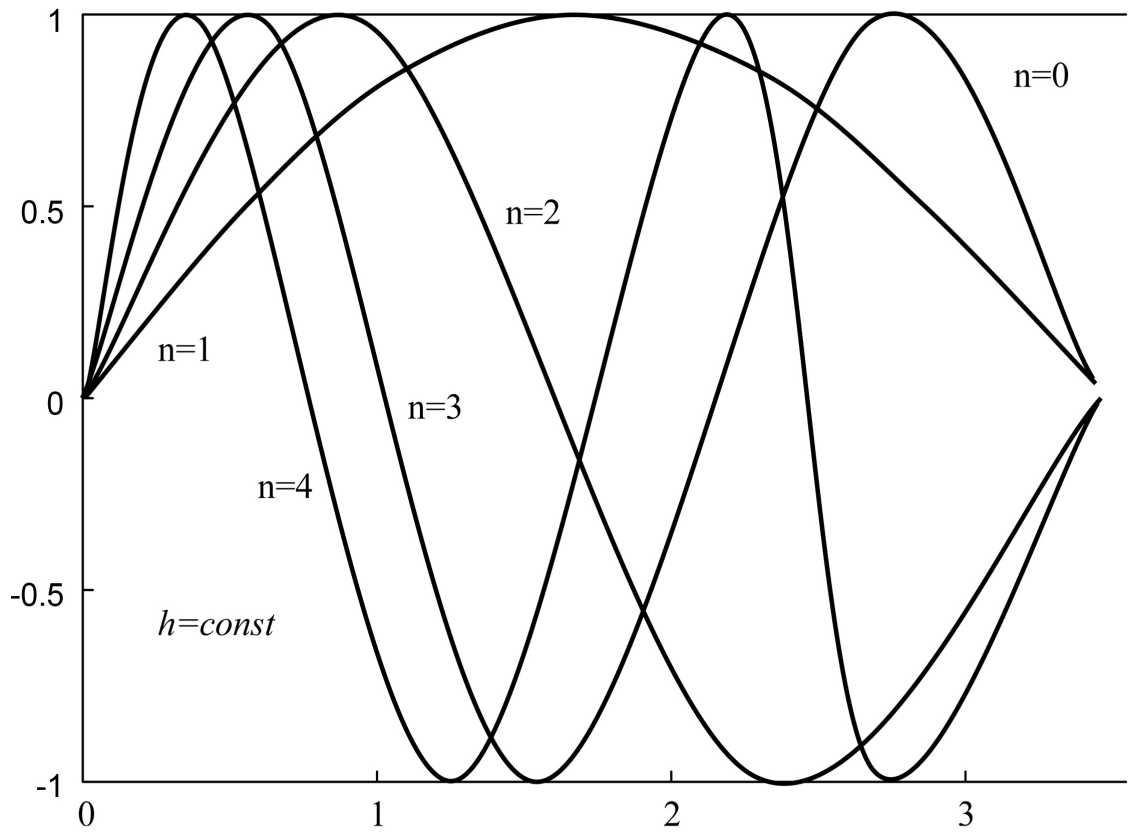

Figure 4. Form for the higher oscillation modes. 
We proceed to the propagation of flexural waves in a symmetric band Kirchhoff-Love of variable thickness. Let us first consider a waveguide with a linear thickness change, presented in Figure 6 and Figure 7 which are free edges. Figure 8 shows the dispersion curves for the first mode, depending on the verge of tilt angle $\varphi / 2$. Curve I corresponds to a strip of constant thickness $h_{0}=h_{1}$. Curve 2 corresponds to a waveguide with an angle of inclination of faces $\varphi / 2=\pi / 4$ or $\operatorname{tg} \varphi / 2=1$ and curve 3 corresponds to a waveguide $\operatorname{tg} \varphi / 2=0.2$. The figure shows that, unlike the bands in the case of constant cross-section of the waveguide with a small tapered angle at the base of the wedge $\alpha$ (Curve 3 ) there exists a finite limit of the phase velocity of the fashion spread, and

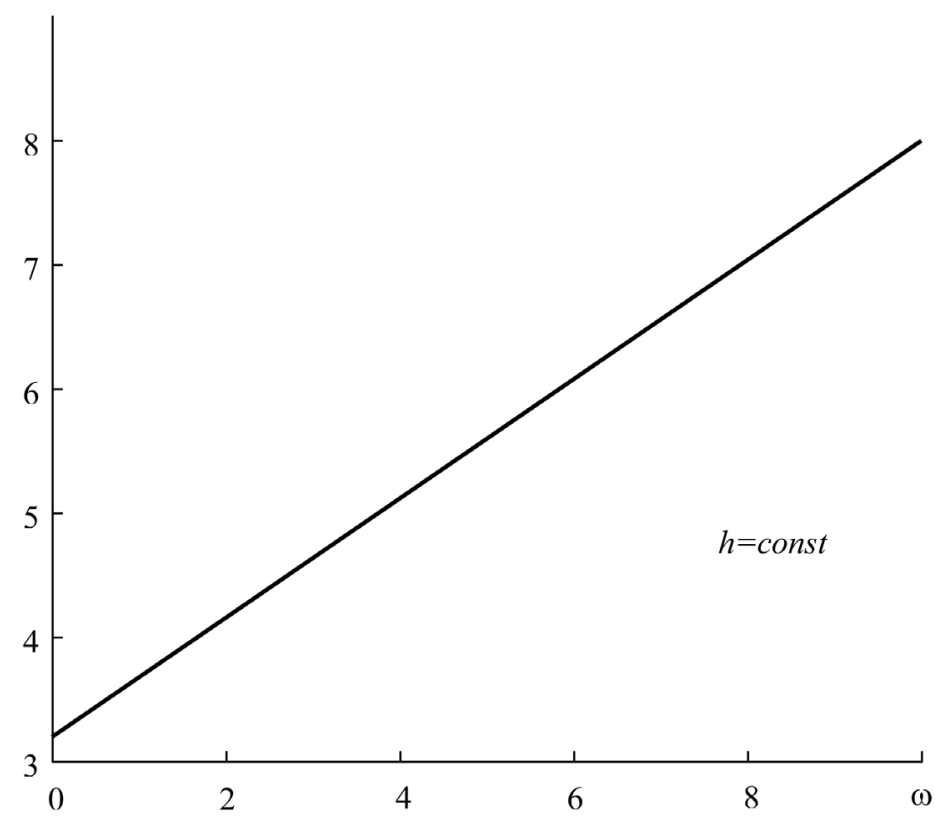

Figure 5. The amplitude of the excited mode depending on the frequency.

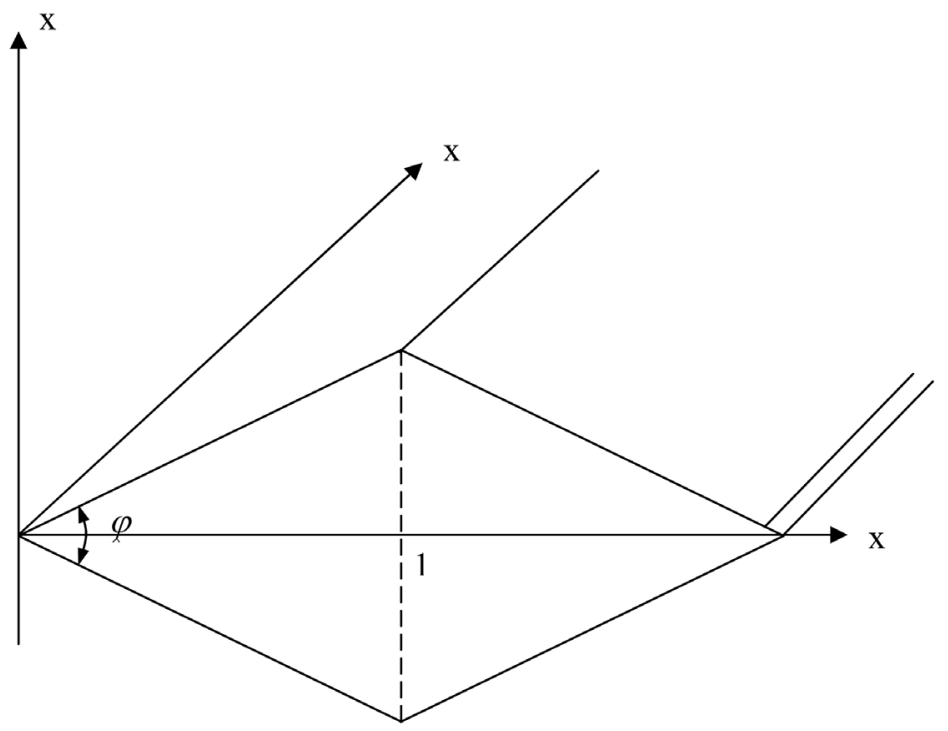

Figure 6. The settlement scheme. 


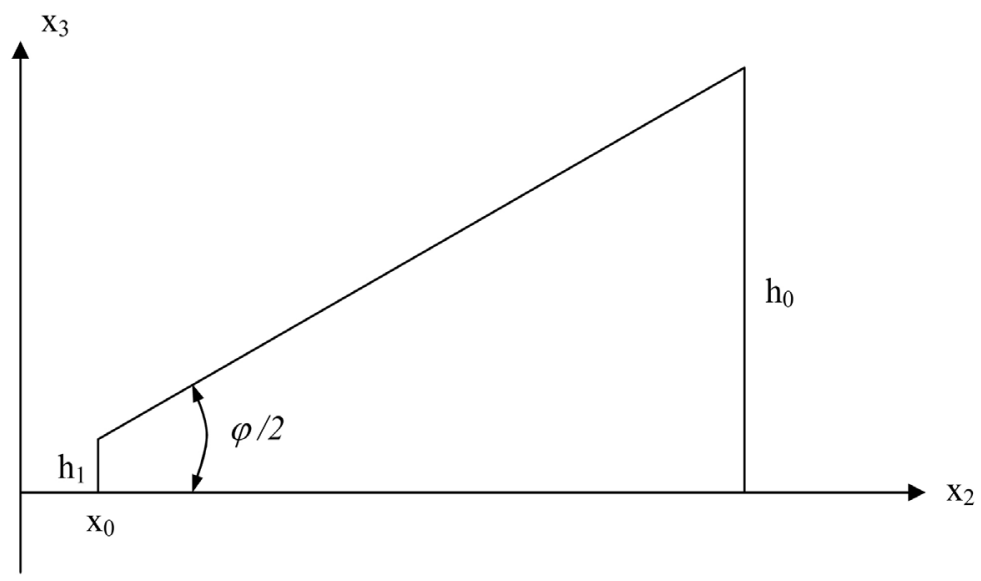

Figure 7. The settlement scheme.

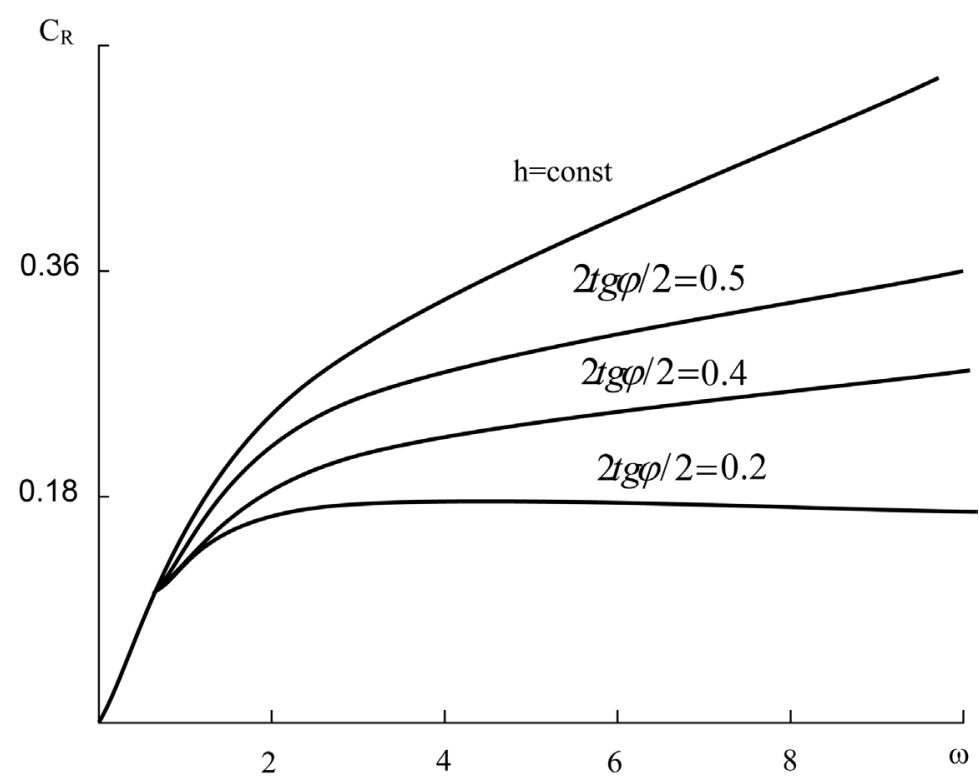

Figure 8. The dependence of the real and imaginary parts of the phase velocity on frequency.

$$
\lim _{\omega \rightarrow \infty} \tilde{C}_{f}=2 C_{s} \operatorname{tg} \frac{\varphi}{2}
$$

where $C_{s}$-The speed of shear waves, which coincides with the results of other studies [11] [12]. Thus, it is shown that-Lava Kirchhoff theory provides a wave propagating in the waveguide is tapered with a sufficiently small angle at the base of the wedge-speed, lower shear wave velocity and different from the Rayleigh wave velocity. Moreover, these waves from a frequency distributed without dispersion. This wave is called "wave Troyanovskiy-Safarov" [13] [14].

Figure 9 shows the waveform of the same frequency for $\omega=10$, from which it follows that the strip of constant thickness behaves like a rod while at the wedge-shaped strip there is a significant localization of waves in the area of acute viburnum, and the more, the smaller the angle $\varphi$. The above fact explains the 


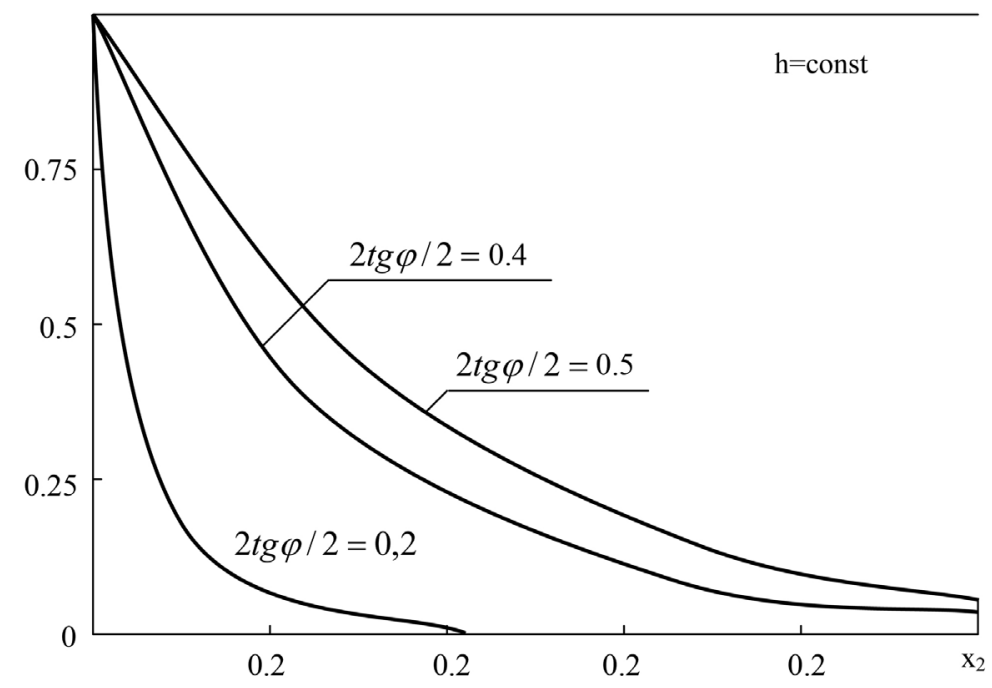

Figure 9. The forms of the coordinate fluctuations $x_{2}$.

Kirchhoff theory-Lava applicability for studying wave propagation in waveguides is tapered, as the frequency increases with decreasing length of one side of the wave modes, with different wave localizes with the sharp edge of the wedge so that the ratio of the wavelength and the effective thickness of the material is in the field of applicability of the theory [15]. This statement is true, the smaller the angle at the base of the wedge.

It should also be noted that the numerical analysis of the dispersion Equation (33) does not allow to show the presence of strictly limit the speed of wave propagation modes, since the computer cannot handle infinitely large quantities. We can only speak about the numerical stability result in a large frequency range, which is confirmed by research. For example, when $\operatorname{tg} \varphi / 2=0.2$ value of the phase velocity of a measured without shear wave velocity at $\omega=3$ and $\omega=$ 40 It differs fifth sign that corresponds to the accuracy of calculations, resulting in test problem.

In the example $h_{0}=0.0001$, it certainly gives an increase of the phase velocity when the frequency increases further, since such a strong localization of the wave to the thin edge of the wedge, starts to affect the characteristic dimension-the thickness of the truncated wedge, and Kirchhoff hypothesis-Lava stops working. To solve the problem of acute wedge numerically is not possible, since the dispersion equation contains a term $D^{-1}$, and the thickness tends to zero flexural rigidity $D$ behaves as a cube and the thickness goes to zero. This significantly increases the "rigidity" (i.e. the ratio between the small and large coefficient) system, increases dramatically the computing time and decreases the accuracy of the results. However, it is clear that you can trust the results obtained where the agreed parameters $h_{0}$ and $\alpha$. We note also that the numerical experiment showed no significant dependence of the phase velocity of the first mode of the Poisson's ratio $v$, and the fact that a family of dispersion curves with different apex angles of the wedge have a similarity property: the ratio of the phase 
velocity to the limit does not depend on the angle of the wedge $\varphi$. On the modes, starting from the second, the speed limit dependence on Poisson's ratio becomes noticeable about $8.5 \%$ for the second mode when changing $0 \leq v \leq 0.5$. Generally, the limit speed increases with the stronger and the more the mode number.

Figure 10 shows the dispersion curves for the first modal wedge $\operatorname{tg} \varphi / 2=0.2$. The figure shows that the speed of the first mode (curve I) is equal to zero for $\omega=0$ and since the frequency $\omega=1$, virtually unchanged. The speed of the second mode (curve 2) is nonzero and finite for $\omega=0$ and stabilized at $\omega=3$. The rest of the modes (curve number matches the number of fashion) have a cut-off frequency, which can be easily determined from Figure 11 (the dependence of the wave number $\alpha$ of the frequency), and decreasing, stabilized (seen 3 and 4 modes) at top speed.

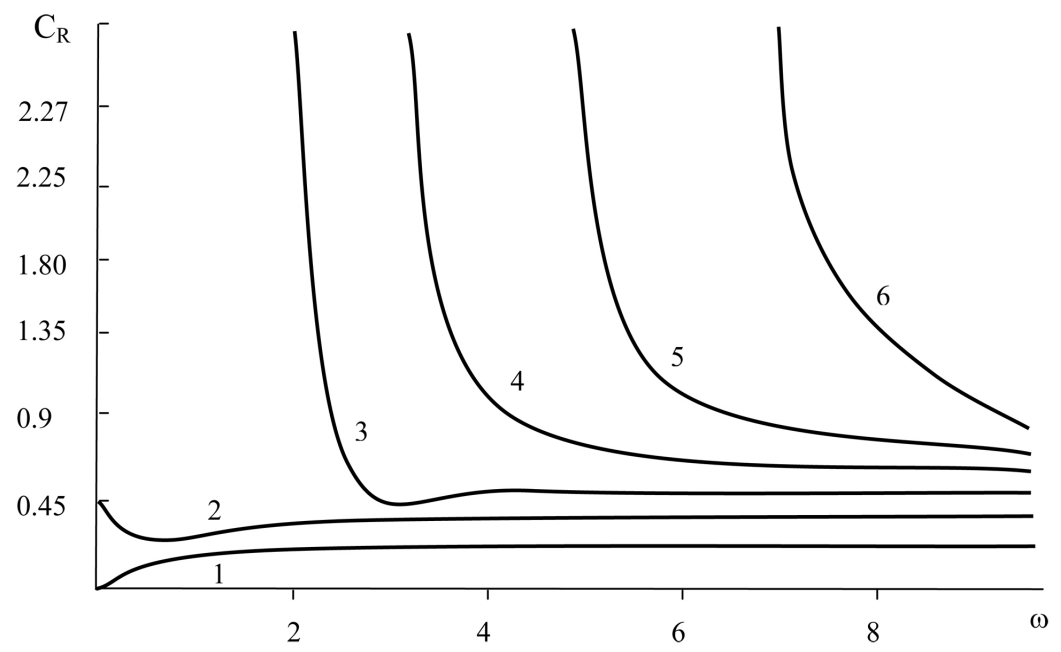

Figure 10. Dependence of the real and imaginary parts of the phase velocity on frequency.

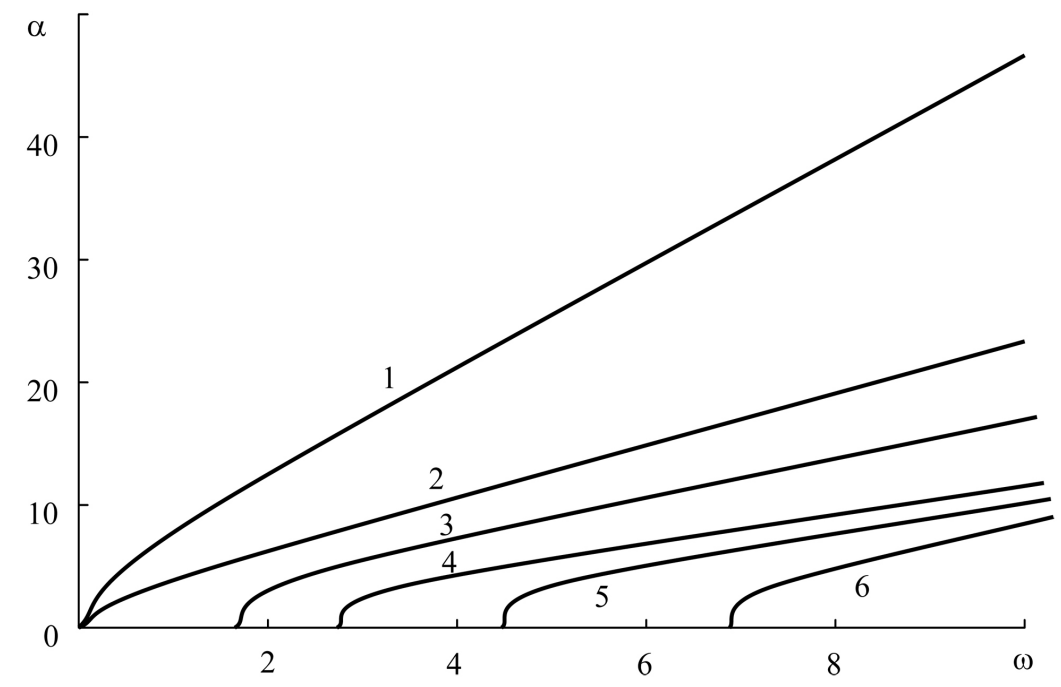

Figure 11. Dependence of the frequency of the wave. 
Figure 12 shows the evolution of the first waveform with the frequency $\omega$ for frequencies $\omega=0.5 ; 1 ; 5$ и 20. Pronounced localized form with increasing frequency. Figures 13-16 show the own forms respectively for $2-4$ modes of vibration for different frequencies: $\omega=1,2,3$ and 4 (the number of grid points corresponds to the number form). And here there are localized forms in the area of thin wedge edge. Figure 16 gives an idea of the degree of localization of the forms at the frequency $\omega=1$, obviously, the lower the number of forms, the stronger it is localized at the edge of the wedge.

Figures 17-19 show the spectral curves of the first three events in the case of the nonlinear dependence of the thickness of the strip from the coordinates $x_{2}$.

$$
h\left(x_{2}\right)=h_{0}+h x_{2}^{p}, \quad 0<x_{2} \leq 1,
$$

where the parameter $\rho$ It was assumed to be $1.5 ; 2 ; 2.5 ; 3$ (curves $1,2,3,4$, respectively, curve " 0 " corresponds to $p=1$-linear relationship).

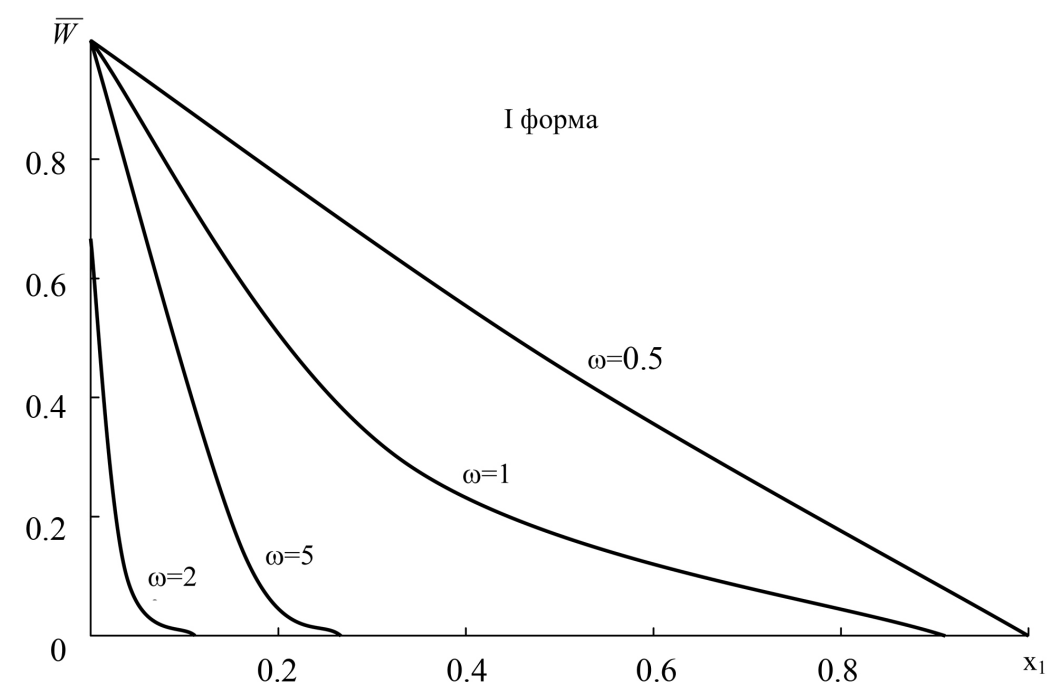

Figure 12. Changing the shape of the coordinate fluctuations $X_{1}$.

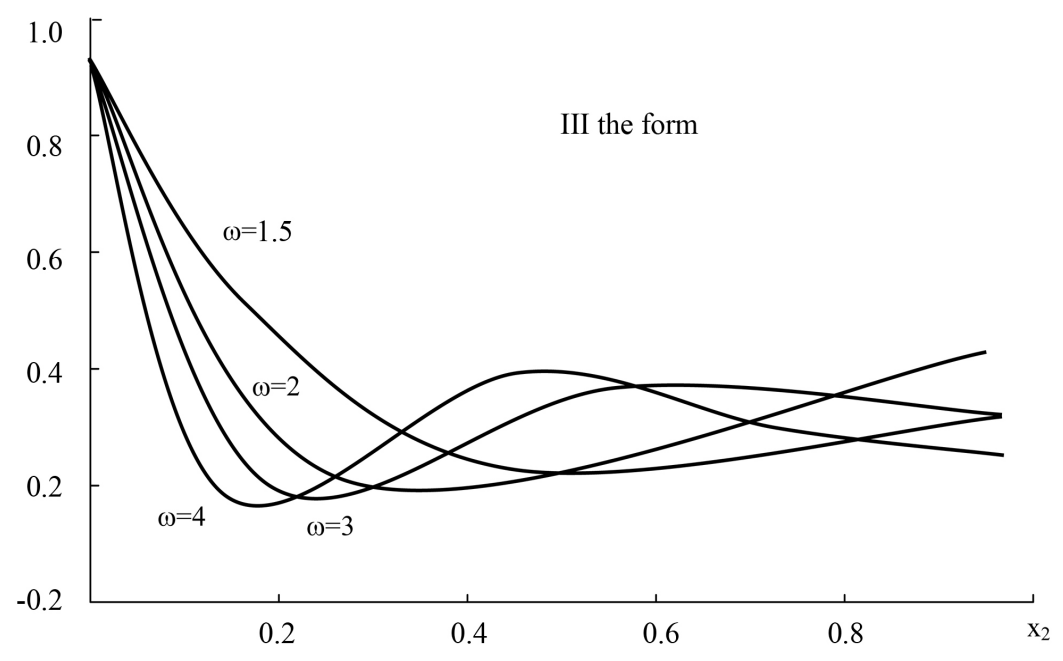

Figure 13. Changing the shape of the coordinate fluctuations $x_{2}$. 


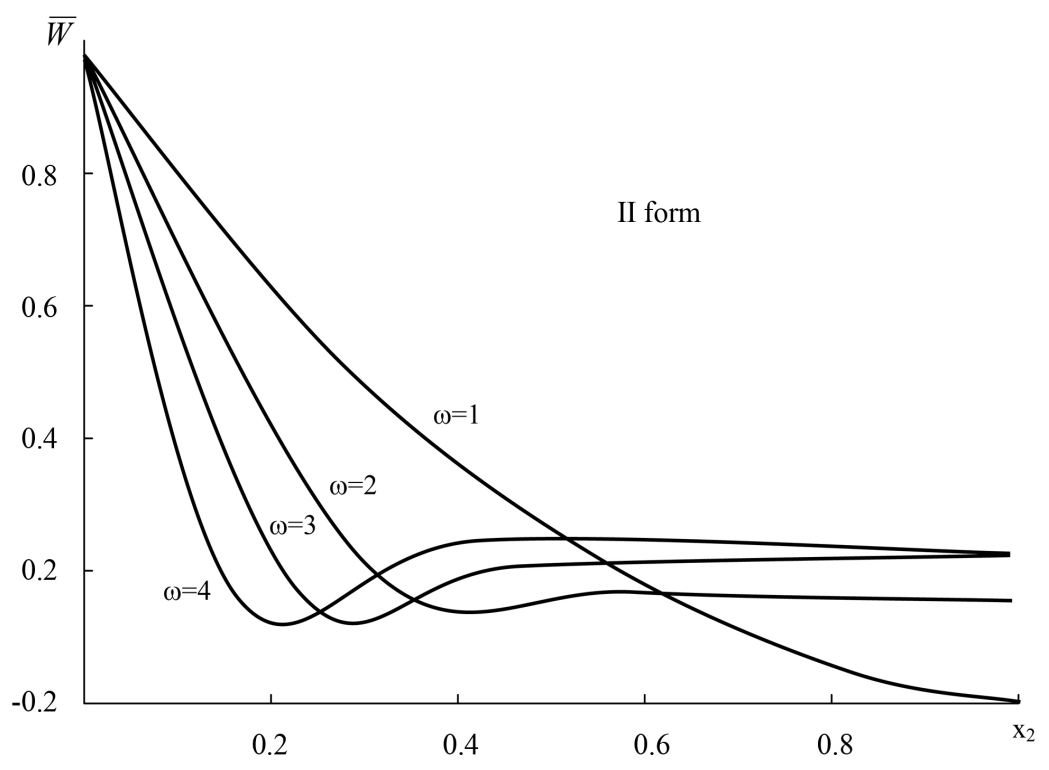

Figure 14. Changing the shape of the coordinate fluctuations.

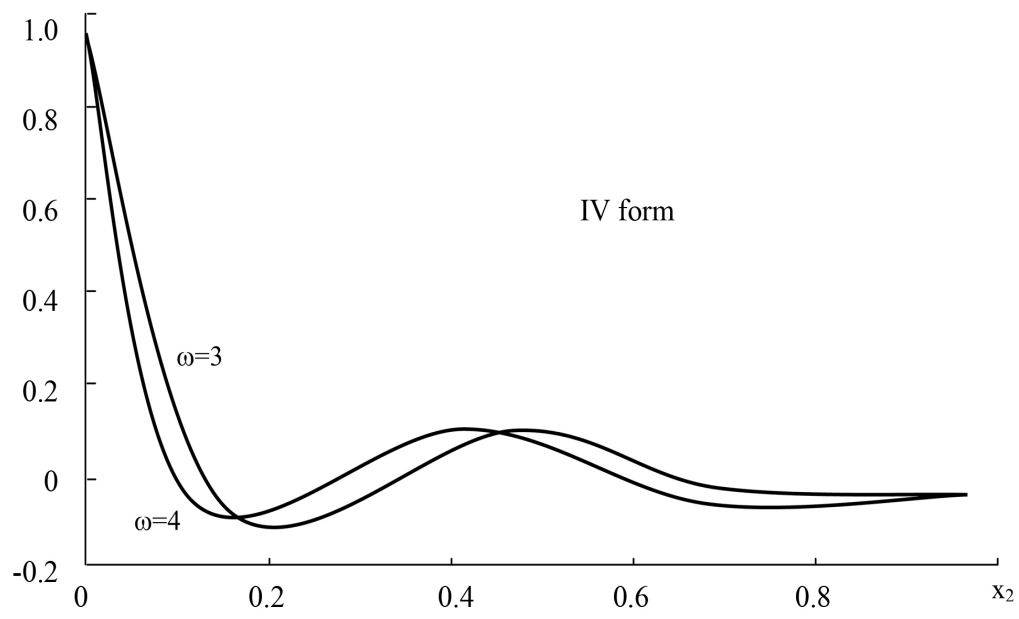

Figure 15. Changing the shape of the coordinate fluctuations.

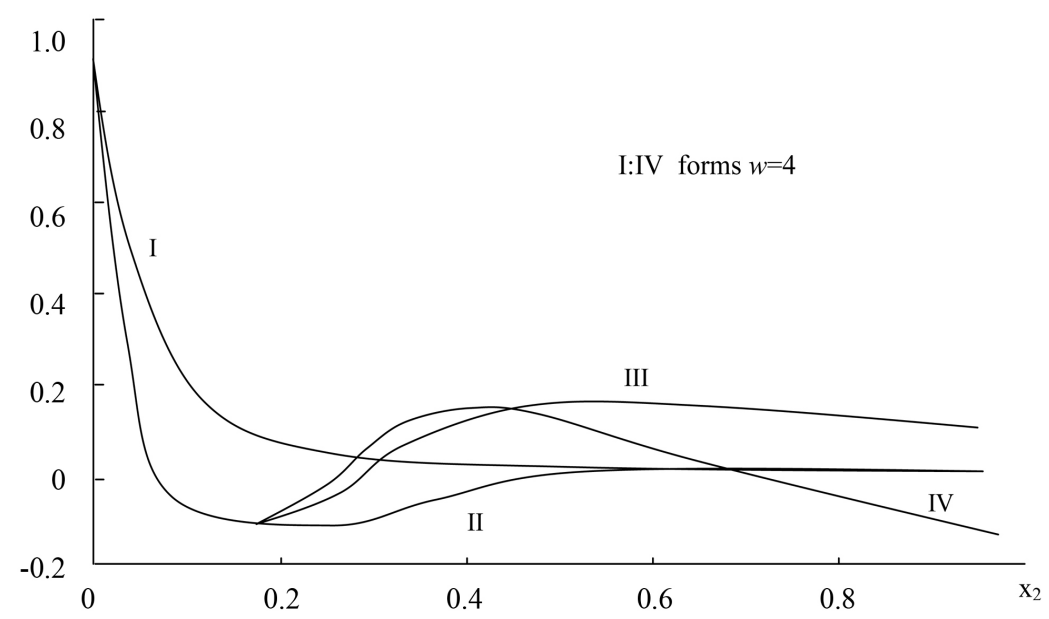

Figure 16. Changing the shape of the coordinate fluctuations. 


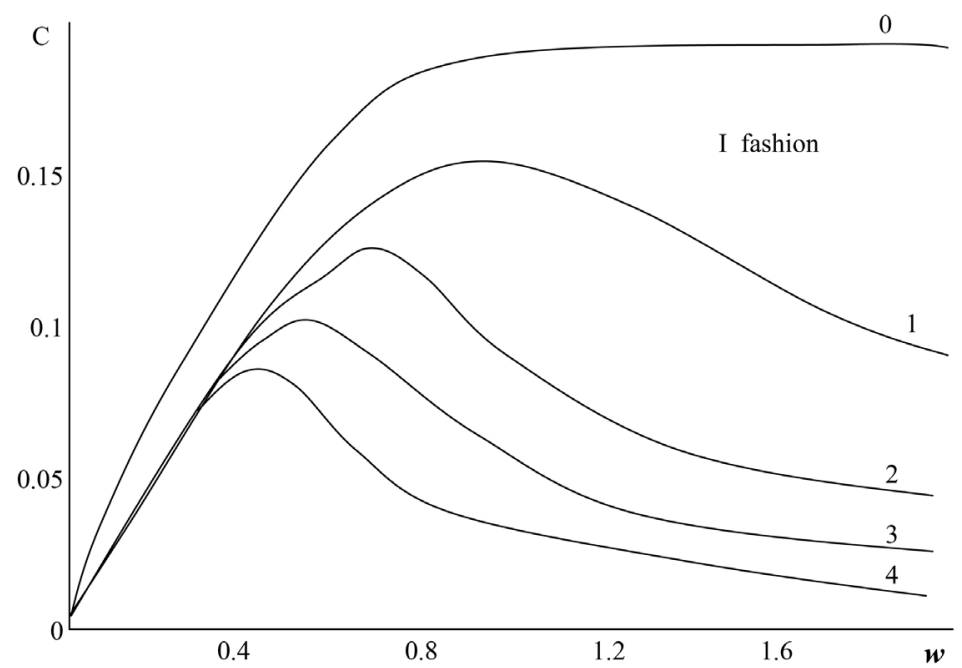

Figure 17. Changing the phase velocity as a function of frequency.

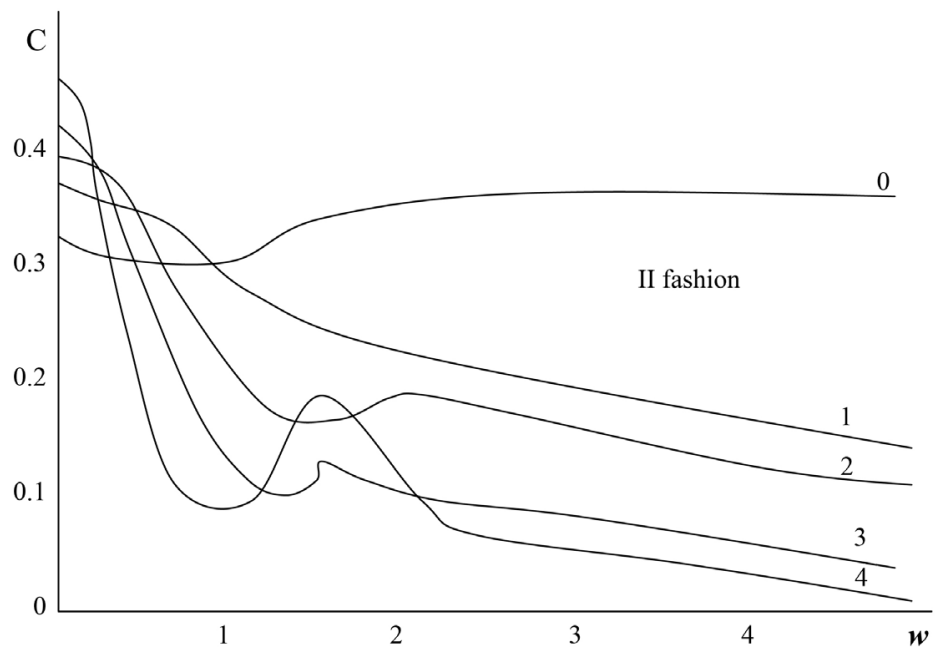

Figure 18. Changing the phase velocity as a function of frequency.

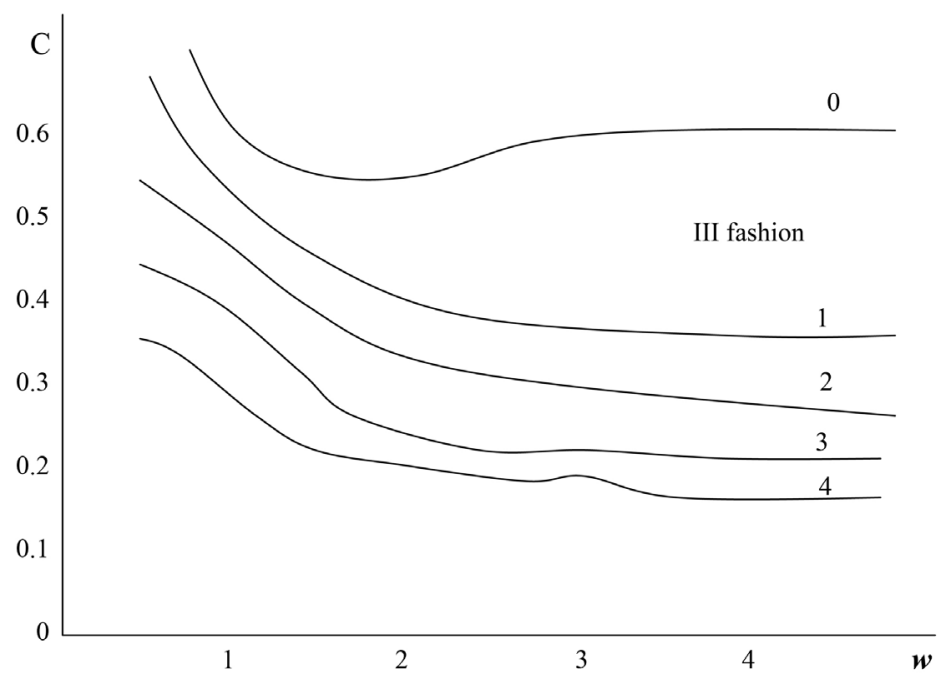

Figure 19. Changing the phase velocity as a function of frequency. 


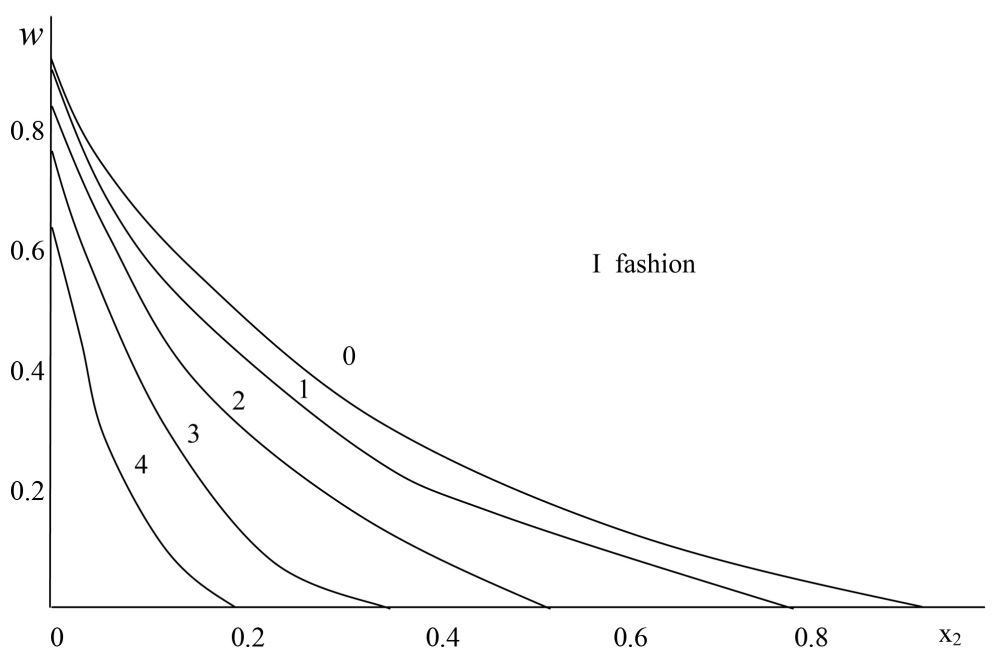

Figure 20. Change in the shape of the plate oscillations along the coordinate.

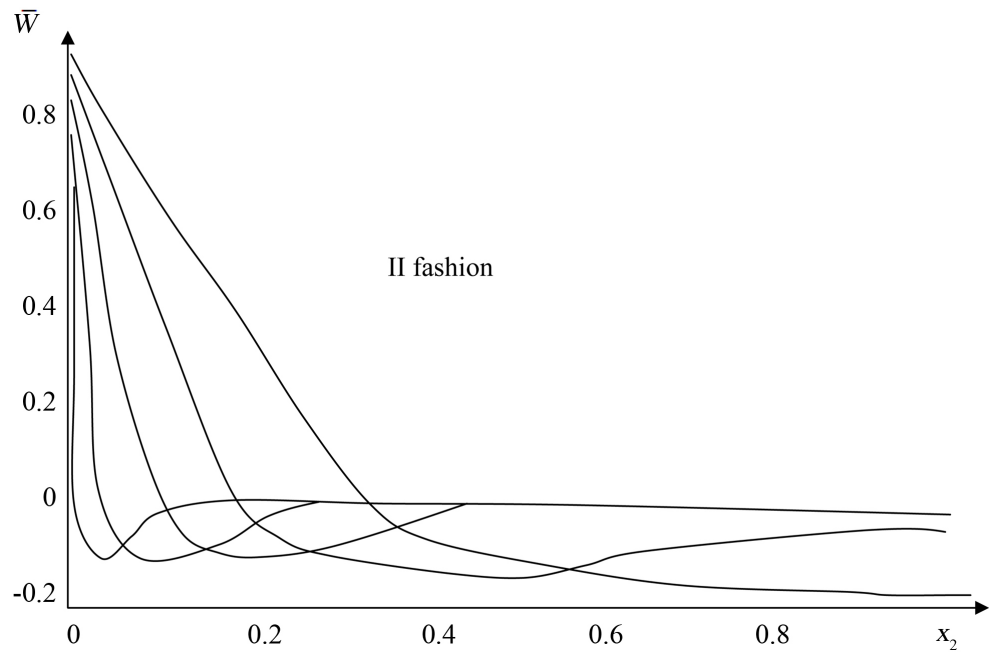

Figure 21. Change in the shape of the plate oscillations along the coordinate.

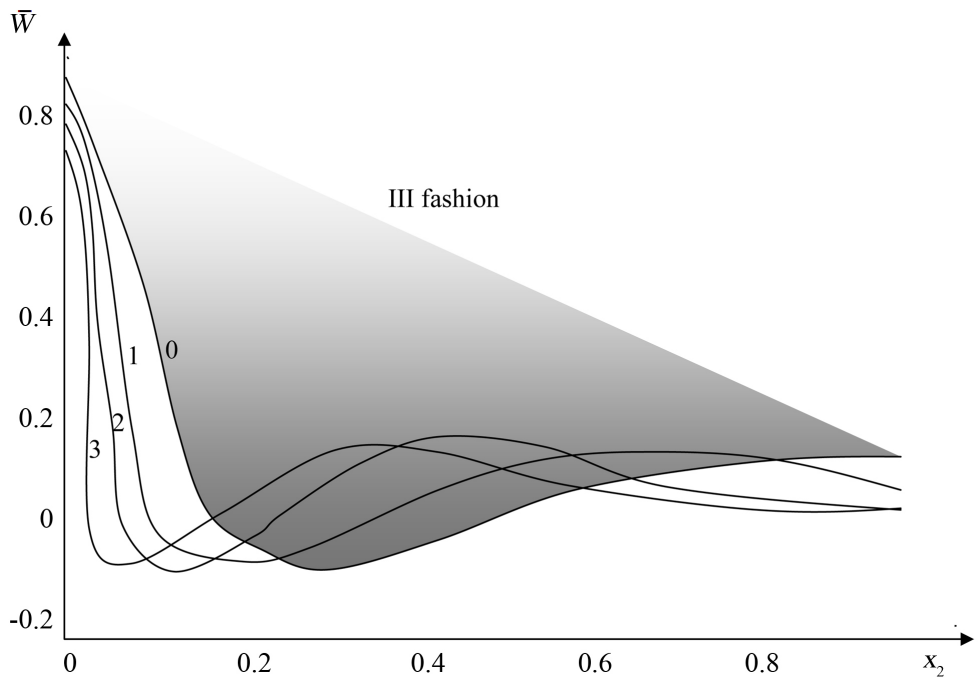

Figure 22. Change in the shape of the oscillations along the coordinate. 
From the equation of " 0 " with the remaining curve shows that they are located on the horizontal high-frequency asymptote, monotonically to zero. The midrange is observed a characteristic peak which is shifted to lower frequencies with an increase in " $p$ ". In accordance with the charts of waveforms at Figures 20-22 quicker and localization of motion near the edge of the waveguide.

Thus, it can be concluded that the phase velocity of the wave in the localized waveguide edge is defined as the frequency increases the rate of change of thickness in the vicinity of the sharp edge.

Figures 23-28 illustrate the solution of the stationary problem for a wedge-shaped waveguide with a linear change in the thickness of the coordinates $x_{2}$ depending on the location of the excitation zone, from which it is clear that

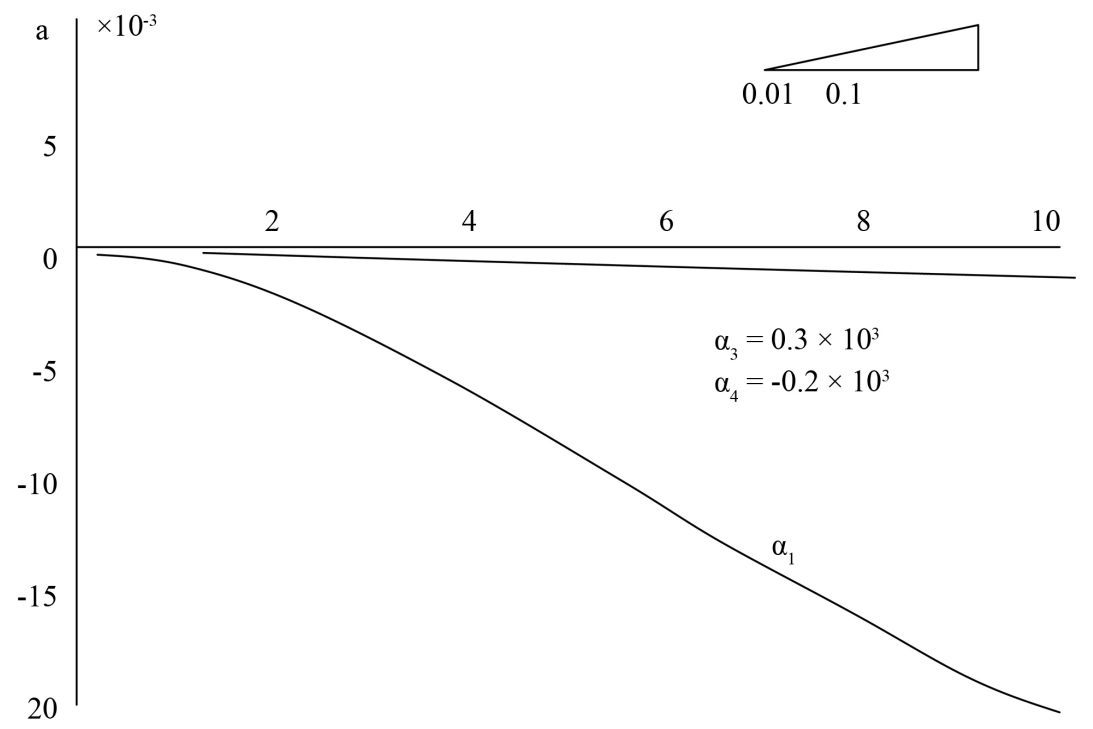

Figure 23. The change factor a depending on the frequency.

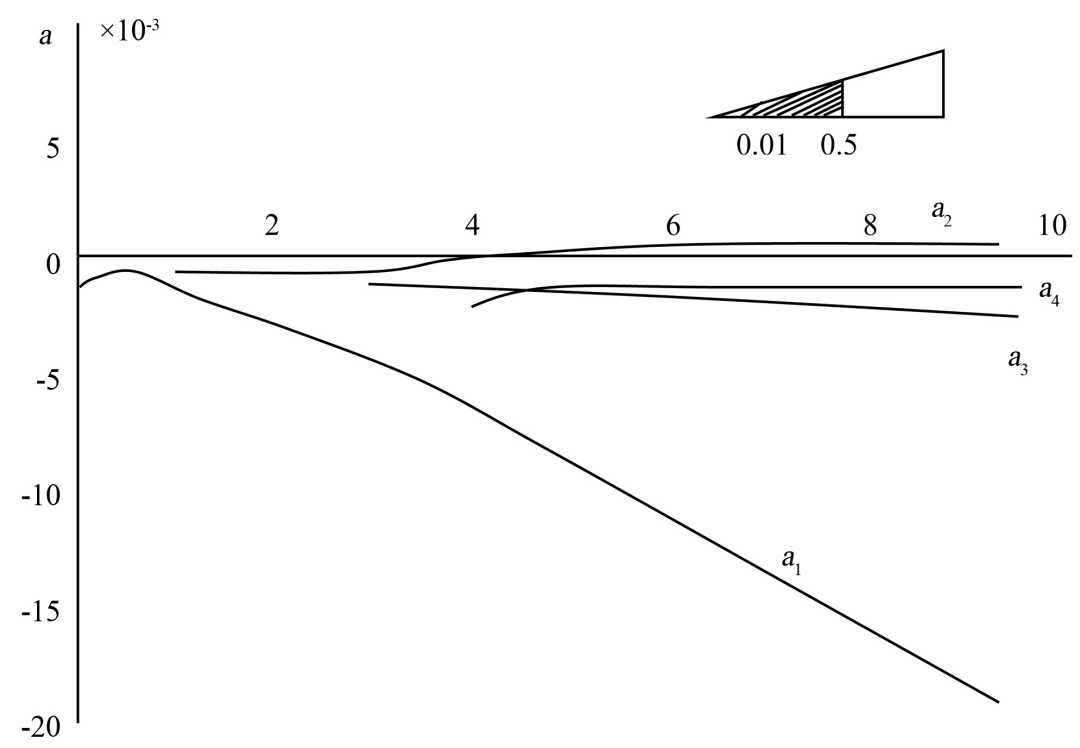

Figure 24. The change factor a depending on the frequency. 


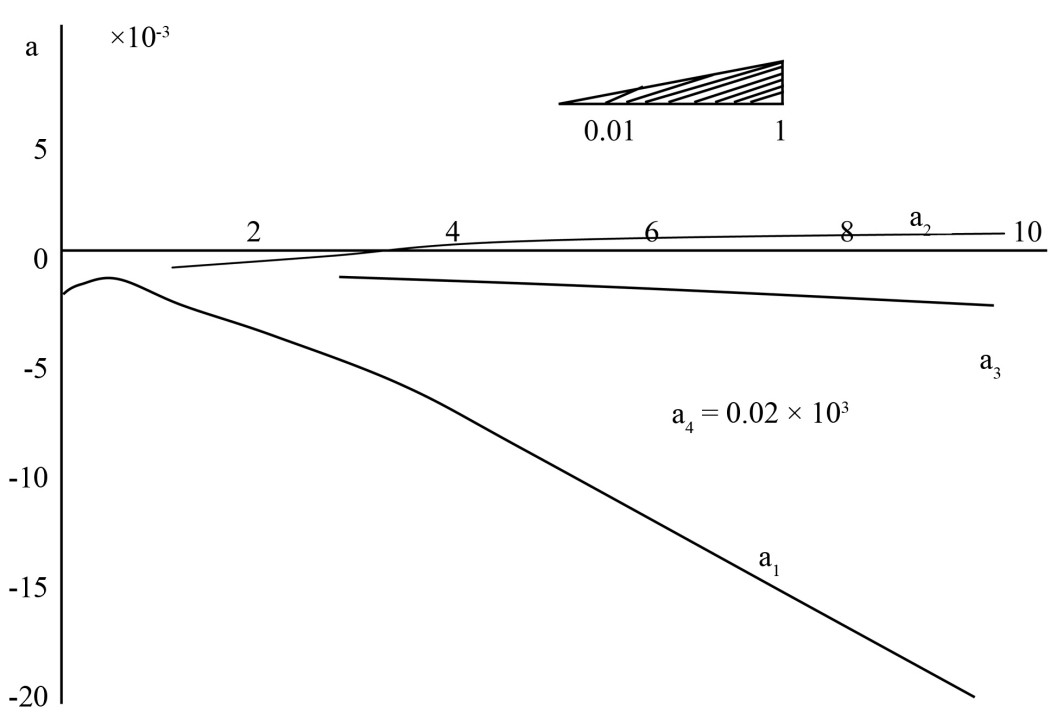

Figure 25. The change factor a depending on the frequency.

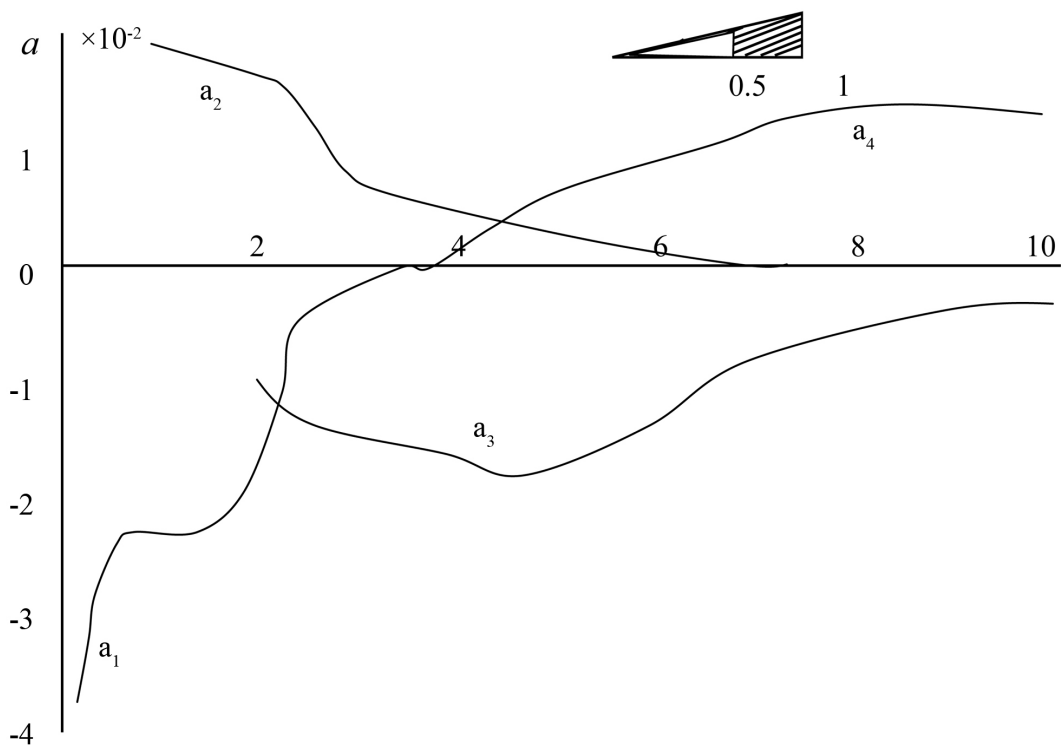

Figure 26. The change factor a depending on the frequency.

the main contribution to the resulting solution brings a sharp edge excited waveguides. Analysis of Figures 23-25 shows that, if aroused sharp edge of the wedge is raised mostly first oscillation mode, and ratio $\alpha_{1}$ increases with increasing frequency.

The amplitude of the remaining modes is not more than 5\% from the first $(\omega=10)$. Upon excitation of the central waveguide portion (Figure 26 and Figure 27) the amplitude of oscillation is 20 - 50 times lower than when excited sharp edge and decreases with increasing frequency. Figure 28 shows the factors driving modes when the excitation zone does not capture any region, the center of the waveguide. The oscillation amplitude is also here oscillations 20 - 50 times less than in the first case. 26 - 28 of the drawings can be made and another 


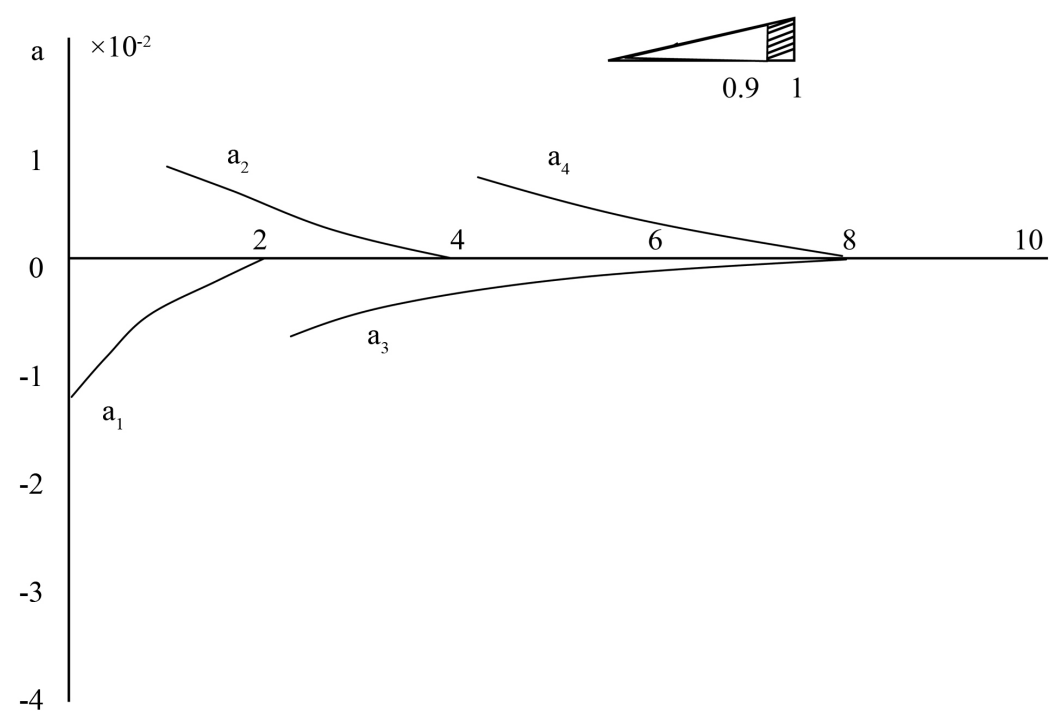

Figure 27. The change factor a depending on the frequency.

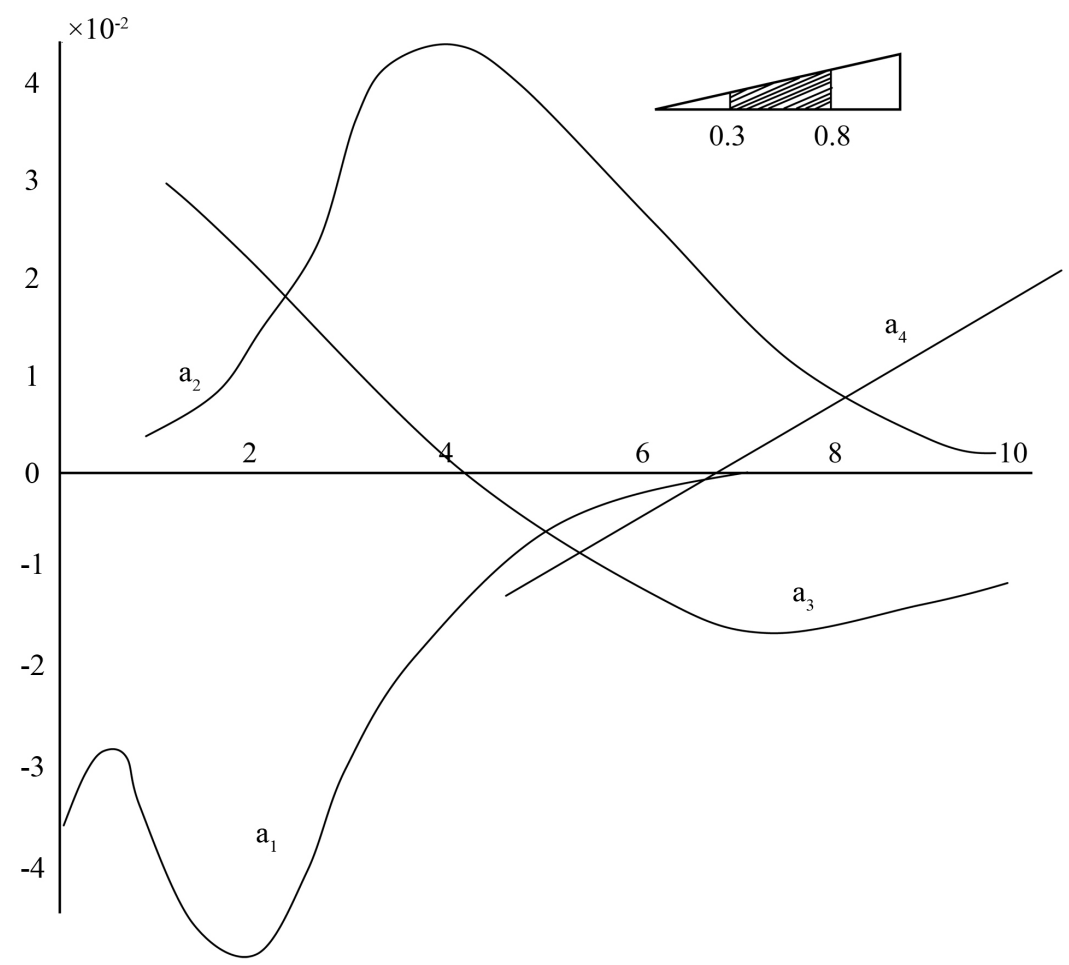

Figure 28. The change factor a depending on the frequency.

conclusion that in this case the entire frequency range can be divided into zones, in which one of the modes propagates mainly. For example, in the case of Figure 25:

$0 \leq \omega \leq 2$ I fashion; $2 \leq \omega \leq 5$ II fashion; $5 \leq \omega \leq 10$ III fashion, t. i.

\section{Conclusions}

On the basis of these results, the followings may be concluded: 
- On the basis of the variation equations of the theory of elasticity, the mathematical formulation of the problem of the propagation of longitudinal waves in plates of variable thickness is reduced to a system of differential equations with the corresponding boundary conditions.

- Showing that the square of the wave number for own endless bands of variable thickness in any combination of the action of the boundary conditions.

- The obtained spectral problem is not self-adjoint, so the associated problem is constructed for it. Coupling system consists of ordinary differential equations with the appropriate boundary conditions. With the help of the Lagrange formula obtained conditions biorthogonality forms. The problem is solved numerically by the method of orthogonal shooting S. K. Godunov in conjunction with the method of Muller.

- Analysis of the data shows that the region with the imaginary theory of Kirchhoff-Love to the plate of constant thickness is limited by the low frequency range. At high frequencies, when wavelength comparable to fashion or less than the thickness of the plate, theory Kirchhoff-Love does not yield reliable results.

- For the phase velocity of propagation modes in the band of variable thickness, there is final repartition unlike the constant cross-section strip.

\section{References}

[1] Bestuzheva, I.P. and Durov, V.N. (1981) On the Propagation of Edge Waves in Elastic Media. Akustik Journal, 27, 487-490.

[2] Bobrovnitsky, Y.I. (1977) Dispersion of Flexural Normal Waves in the Thin Strip. Akustik Journal, 23, 34-40.

[3] Skudrzyk, E. (1971) Simple and Complex Vibratory Systems. University Park and London, $558 \mathrm{p}$.

[4] Grinchenko, V.T. and Myaleshka, V.V. (1981) Harmonic Oscillations and Waves in Elastic Bodies. Kiev, 284 p.

[5] Kravchenko, V.T. and Myaleshka, V.V. (1981) Properties of Harmonic Waves Propagating along the Edges of the Rectangular Elastic Wedge. Akustik Journal, 27, 206-212.

[6] Meeker, T. and Metzler, A. (1966) Ducting in Long Cylinders and Plates. Def. Acoustics, Principles and Methods, Trans. from English, 1 A, 140-203.

[7] Koltunov, M.A. (1976) Creep and Relaxation. High School, Moscow, 273 p.

[8] Rabotnov, Y.N. (1977) Hereditary Elements of Mechanics of Solids. Nauka, Moscow, 384 p.

[9] Neumark, M.A. (1969) Linear Differential Operators. Nauka, Moscow, 526 p.

[10] Mysovskikh, I.P. Lectures on Methods of Calculation: St. Petersburg University. $1998 \mathrm{p}$.

[11] Safarov, I.I., Teshaev, M.H. and Boltaev, Z.I. (2012) Wave Processes in the Mechanical Waveguide. Lambert Academic Publishing, Saarbrücken, 217 p.

[12] Safarov, I.I., Boltaev, Z.I. and Akhmedov, M.S. (2015) Distribution of the Natural Waves. Lambert Academic Publishing, Saarbrücken, 110 p.

[13] Safarov, I.I., Akhmedov, M.S. and Rajabov, O. (2015) Vibrations of Plates and Shells 
with Attached Concentrated Mass. Lambert Academic Publishing, Saarbrücken, 92 p.

[14] Safarov, I.I., Akhmedov, M.S. and Boltayev, Z.I. (2015) Dissemination Sinusoidal Waves in of a Viscoelastic Strip. Global Journal of Science Frontier Research: F Mathematics and Decision Sciences, 15, 39-60.

[15] Bazarov, M.B., Safarov, I.I., Shokin, Y.I. (1996) Numerical Simulation of Oscillations of Dissipatively Homogeneous and Inhomogeneous Mechanical Systems. Novosibirsk, $187 \mathrm{p}$. 